\title{
RANDOM EVOLUTIONARY DYNAMICS DRIVEN BY FITNESS AND HOUSE-OF-CARDS MUTATIONS. SAMPLING FORMULAE
}

\author{
THIERRY E. HUILLET
}

\begin{abstract}
We first revisit the multi-allelic mutation-fitness balance problem, especially when mutations obey a house of cards condition, where the discrete-time deterministic evolutionary dynamics of the allelic frequencies derives from a Shahshahani potential. We then consider multi-allelic WrightFisher stochastic models whose deviation to neutrality is from the Shahshahani mutation/selection potential. We next focus on the weak selection, weak mutation cases and, making use of a Gamma calculus, we compute the normalizing partition functions of the invariant probability densities appearing in their Wright-Fisher diffusive approximations. Using these results, Generalized Ewens sampling formulae (ESF) from the equilibrium distributions are derived. We start treating the ESF in the mixed mutation/selection potential case and then we restrict ourselves to the ESF in the simpler house-of-cards mutations only situation. We also address some issues concerning sampling problems from infinitely-many alleles weak limits.
\end{abstract}

Keywords: Evolutionary genetics, fitness landscape, house-of-cards mutations, Shahshahani gradient, Wright-Fisher random genetic drift, Gamma calculus, generalized Ewens sampling formulae.

\section{INTRODUCTION}

Population genetics is concerned with the fate of multi-allelic population frequencies when various driving 'forces' such as selection or mutation are involved. We will briefly revisit the basics of the deterministic dynamics arising in discrete-time asexual evolutionary genetics when the origin of motion is either fitness or mutations or both. We will mostly consider the multi-allelic diploid case under the Hardy-Weinberg hypothesis. Firstly, we will consider evolution under general fitness mechanisms (Section 2), then we deal with general mutation mechanisms (Section 3). Some particular fitness/mutation patterns are discussed in the process. In some cases, the dynamics driven by fitness only takes the form of a Shahshahani gradient dynamics, as deriving from a Shahshahani selection potential, [30, [5]. In Section 4 , we give one way to combine the fitness and the mutation effects. All these issues are of course part of the standard models discussed for example in [8], [2, 21], 22, [26] and 20]. We then focus on a reversible mutation pattern in which mutation probabilities between any two states only depend on the target state (the house of cards condition for mutations). When combined with selection effects, the dynamics driven both by selection and house-of-cards mutations takes the form of a Shahshahani gradient-like dynamics with drift deriving from a Shahshahani potential mixing additively the mutation and selection potentials, [15. 
On top of such deterministic dynamics, we add (Section 5) a random genetic drift while considering a multi-allelic Wright-Fisher Markov chain whose deviation to neutrality appears as a drift deriving from the Shahshahani mutation/selection potential. Some scalings brings this Markov chain into multi-allelic Wright-Fisher diffusion processes with a unique invariant probability density describing the joint allelic frequencies at equilibrium, 32. We consider the weak selection, weak mutation cases and we compute the normalizing partition functions of the corresponding invariant densities.

Generalized Ewens sampling formulae (ESF) from such equilibrium allelic frequencies are then desirable and these are obtained in Section 6; they make use of the explicit expressions of the partition functions just introduced. Such ancient and long-standing questions go back to [37]; see [34] and [25]. We start treating the ESF in the mixed mutation/selection potential case (Section 6.1) and then we restrict ourselves to the ESF in the simpler house-of-cards mutations only context (Section 6.2). We show that such problems are amenable to the evaluation of some functionals of skew-symmetric Dirichlet distributed random variables (rvs) and for this purpose, we make extensive use of a 'Gamma-calculus' precisely designed to evaluate such functionals. We also address some issues concerning the availability of sampling formulas in some infinitely-many alleles weak limits.

\section{Evolution DRIVEn By Fitness: THE Deterministic POINT OF VIEW}

2.1. Single locus: the case of a diploid population with $K$ alleles. We briefly describe the frequency distribution dynamics when fitness (or selection) only drives the process. We consider diploid populations.

2.1.1. Joint evolutionary dynamics. Consider $K$ alleles $A_{k}, k=1, \ldots, K$ and let $A_{k} A_{l}$ be the genotypes attached to a single locus. Let $w_{k, l} \in[0,1], k, l=1, \ldots, K$ stand for the absolute fitness of the genotypes $A_{k} A_{l}$. We shall assume $w_{k, l}=w_{l, k}$ ( $w_{k, l}$ being the probability of an $A_{k} A_{l}$ surviving to maturity, it is natural to take $\left.w_{k, l}=w_{l, k}\right)$. Let then $W$ be the symmetric fitness matrix with $k, l$-entry $w_{k, l}$.

Assume the current frequency distribution at generation $r \in\{0,1,2, \ldots\}$ of the genotypes $A_{k} A_{l}$ is given by $x_{k, l}$. Let $X$ be the frequencies array with $k, l$-entry $x_{k, l}$, obeying $\sum_{k, l} x_{k, l}=1$. The joint evolutionary dynamics in the diploid case is given by the updating]:

$$
x_{k, l}^{+}=x_{k, l} \frac{w_{k, l}}{\omega(X)} \text { where } \omega(X)=\sum_{k, l} x_{k, l} w_{k, l},
$$

where the relative fitness of the genotype $A_{k} A_{l}$ is $w_{k, l} / \omega(X)$. The joint dynamics takes on the matrix form:

$$
X^{+}=\frac{1}{\omega(X)} X \circ W=\frac{1}{\omega(X)} W \circ X
$$

where $\circ$ stands for the (commutative) Hadamard product of matrices.

\footnotetext{
${ }^{1}$ The symbol + is a common and useful notation to denote the updated frequency from generation $r$ to $r+1$.
} 
With 1 a column-vector of ones, $\mathbf{1}^{\prime}$ its transpose and $J=\mathbf{1 1}^{\prime}$ the $K \times K$ matrix whose entries are all 1 (the identity for $\circ)^{2}$, then

$$
\Delta X:=X^{+}-X=\frac{1}{\omega(X)}(W-\omega(X) J) \circ X=\frac{1}{\omega(X)} X \circ(W-\omega(X) J) .
$$

Let

$$
\sigma^{2}(X)=\sum_{k, l=1}^{K} x_{k, l}\left(w_{k, l}-\omega(X)\right)^{2} ; \bar{\sigma}^{2}(X)=\sum_{k, l=1}^{K} x_{k, l}\left(\frac{w_{k, l}}{\omega(X)}-1\right)^{2}=\frac{\sigma^{2}(X)}{\omega(X)^{2}}
$$

stand respectively for the genotypic variance in absolute fitness and the diploid variance in relative fitness. Note that, owing to $\sum_{k, l} x_{k, l}=1$ and $\omega(X)=\sum_{k, l} x_{k, l} w_{k, l}$,

$$
\omega(X) \bar{\sigma}^{2}(X)=\omega(X) \sum_{k, l=1}^{K} x_{k, l}\left(\frac{w_{k, l}}{\omega(X)}-1\right)^{2}=\frac{1}{\omega(X)} \sum_{k, l} x_{k, l} w_{k, l}^{2}-\omega(X) .
$$

The partial increase of the mean fitness (where the change of mean fitness only comes through changes in the frequencies $x_{k, l}$ ) is given by

$$
\delta \omega(X):=\sum_{k, l} \Delta x_{k, l} w_{k, l}=\sum_{k, l} x_{k, l}\left(\frac{w_{k, l}^{2}}{\omega(X)}-w_{k, l}\right)=\omega(X) \bar{\sigma}^{2}(X)>0,
$$

with a relative rate of increase: $\delta \omega(X) / \omega(X)=\bar{\sigma}^{2}(X)$. The latter result (3) constitutes the diploid version of the 1930 Fisher fundamental theorem of natural selection for asexual populations; see ([8], Sections $(2.8,2.9), 28]$ ) and [27]) for a deeper insight on its meaning.

2.1.2. Marginal multi-allelic dynamics. Assuming a Hardy-Weinberg equilibrium, the frequency distribution at generation $r$, say $x_{k, l}$, of the genotypes $A_{k} A_{l}$ is given by: $x_{k, l}=x_{k} x_{l}$ where $x_{k}=\sum_{l} x_{k, l}$ is the marginal frequency of type- $k$ allele $A_{k}$ in a genotypic population. The marginal allelic frequency vector is $\mathbf{x}=X \mathbf{1}$ ( $\mathbf{1}$ is the unit $K$-column-vector) and the mean allelic fitness is given by the quadratic form: $\omega(\mathbf{x}):=\sum_{k, l} x_{k} x_{l} w_{k, l}=\mathbf{x}^{\prime} W \mathbf{x}$. The mean fitness $\omega(\mathbf{x})=\mathbf{x}^{\prime} W \mathbf{x}$ is homogeneous of degree $d=2$ in the variables $\mathbf{x}$. Let

$$
\sigma^{2}(\mathbf{x})=\sum_{k, l=1}^{K} x_{k} x_{l}\left(w_{k, l}-\omega(\mathbf{x})\right)^{2} ; \bar{\sigma}^{2}(\mathbf{x})=\sum_{k, l=1}^{K} x_{k} x_{l}\left(\frac{w_{k, l}}{\omega(\mathbf{x})}-1\right)^{2}=\frac{\sigma^{2}(\mathbf{x})}{\omega(\mathbf{x})^{2}}
$$

be respectively the genotypic variance in absolute fitness and the diploid variance in relative fitness.

If we first define the frequency-dependent marginal fitness of $A_{k}$ by $w_{k}(\mathbf{x})=$ $(W \mathbf{x})_{k}:=\sum_{l} w_{k, l} x_{l}$, the marginal dynamics is given by:

$$
x_{k}^{+}=x_{k} \frac{w_{k}(\mathbf{x})}{\omega(\mathbf{x})}=\frac{1}{\omega(\mathbf{x})} x_{k}(W \mathbf{x})_{k}=: p_{k}(\mathbf{x}), k=1, \ldots, K .
$$

\footnotetext{
${ }^{2}$ In the sequel, a boldface variable, say $\mathbf{x}$, will represent a column-vector so that its transpose, say $\mathbf{x}^{\prime}$, will be a row-vector. Similarly, $A^{\prime}$ will stand for the transpose of some matrix $A$.
} 
Letting $D_{\mathbf{x}}:=\operatorname{diag}\left(x_{k}, k=1, \ldots, K\right)$, if the allelic frequency distribution is summarized in the column-vector $\mathbf{x}:=x_{k}, k=1, \ldots, K$, (5) reads in vector form 3

$$
\mathbf{x}^{+}=\frac{1}{\omega(\mathbf{x})} D_{\mathbf{x}} W \mathbf{x}=\frac{1}{\omega(\mathbf{x})} D_{W \mathbf{x}} \mathbf{x}=: \mathbf{p}(\mathbf{x}),
$$

where $\mathbf{p}^{\prime}:=\left(p_{1}, \ldots, p_{K}\right)$ maps the $(K-1)$-dimensional simplex

$$
\mathcal{S}_{K}:=\left\{\mathbf{x} \geq \mathbf{0}:|\mathbf{x}|:=\sum_{k=1}^{K} x_{k}=1\right\},
$$

into itself.

\section{Two particular cases:}

(i) The haploid case. Let $\mathbf{w}:=w_{k} \in(0,1], k=1, \ldots, K$, denote the absolute fitnesses of the $K$ alleles and suppose, without loss of generality, that $0<w_{1} \leq$ $\ldots \leq w_{K}=1$ (so that allele $A_{K}$ has largest fitness 1). Let $w(\mathbf{x}):=\sum_{l} w_{l} x_{l}=\mathbf{w}^{\prime} \mathbf{x}$ denote the mean fitness of the population. Plugging $W=\mathbf{w} \mathbf{1}^{\prime}$ in (6), the dynamics (6) boils down to

$$
\mathbf{x}^{+}=\mathbf{p}(\mathbf{x})=\frac{1}{w(\mathbf{x})} D_{\mathbf{w}} \mathbf{x}=\frac{1}{w(\mathbf{x})} D_{\mathbf{x}} \mathbf{w}
$$

giving the update of the frequency distribution of alleles in an haploid population where alleles (and not pairs of alleles) are attached to some locus. Along (7), the absolute mean fitness $w(\mathbf{x})$ increases. Indeed, with $\Delta w(\mathbf{x}):=w\left(\mathbf{x}^{+}\right)-w(\mathbf{x})$ :

$$
\Delta w(\mathbf{x})=\sum_{k} w_{k} \Delta x_{k}=\sum_{k} w_{k} x_{k}\left(\frac{w_{k}}{w(\mathbf{x})}-1\right)=\frac{1}{w(\mathbf{x})}\left(\sum_{k} w_{k}^{2} x_{k}-w(\mathbf{x})^{2}\right) \geq 0,
$$

and it is $>0$ except when $\mathbf{x} \in \mathcal{S}_{K}$ is such that $\left\{k: x_{k}>0\right\} \subseteq\left\{k: w_{k}=1\right\}$, the set of alleles with maximal fitness. Such xs are equilibrium states of (7). In particular, an extremal vector $\mathbf{x}^{\prime}=\mathbf{e}_{k}^{\prime}:=(0, \ldots, 0,1,0, \ldots, 0)$ with $k \in\left\{k: w_{k}=1\right\}$ is a pure (or monomorphic) equilibrium state.

The mean fitness is maximal at equilibrium. The relative rate of increase of $w(\mathbf{x})$ is:

$$
\frac{\Delta w(\mathbf{x})}{w(\mathbf{x})}=\sum_{k} x_{k}\left(\frac{w_{k}}{w(\mathbf{x})}-1\right)^{2}=\sum_{k} \frac{\left(\Delta x_{k}\right)^{2}}{x_{k}}=\bar{\sigma}^{2}(\mathbf{x}),
$$

where $\bar{\sigma}^{2}(\mathbf{x})$ is the variance in relative fitness $\bar{\sigma}^{2}(\mathbf{x})$ given by

$$
\bar{\sigma}^{2}(\mathbf{x})=\sum_{k=1}^{K} x_{k}\left(\frac{w_{k}}{w(\mathbf{x})}-1\right)^{2}=\frac{\sigma^{2}(\mathbf{x})}{w(\mathbf{x})^{2}} .
$$

Thus the population mean fitness is non-decreasing. As a consequence, if there is an unique allele whose fitness strictly dominates the ones of the others, starting from any initial state which is not an extremal point of $\mathcal{S}_{K}$, the haploid trajectories will ultimately converge to this fittest state (survival of the fittest allele).

\footnotetext{
${ }^{3} D_{\mathbf{x}} W \mathbf{x}$ is the Schur product $\mathbf{x} \circ W \mathbf{x}$ of vector $\mathbf{x}$ and vector $W \mathbf{x}$. See 21] page 238 for a similar notational convenience.
} 
(ii) The diploid case with multiplicative fitnesses. Suppose that $w_{k, l}=w_{k} w_{l}$, or in matrix form that $W=\mathbf{w w}^{\prime}$. Then selection acts on the gametes rather than on the genotypes. Observing $\frac{w_{k}(\mathbf{x})}{\mathbf{x}^{\prime} W \mathbf{x}}=\frac{w_{k}}{\sum_{l} w_{l} x_{l}}$, the dynamics (16) boils down to the one (7) of haploid populations. However, the mean fitness in this case is $\omega(\mathbf{x})=\left(\sum_{l} w_{l} x_{l}\right)^{2}$ and not $w(\mathbf{x})=\sum_{l} w_{l} x_{l}$ as in the haploid case.

2.1.3. Increase of mean fitness for diploid populations. Similarly, defining $0 \leq R(\mathbf{x}):=\sum_{k, l} x_{k}\left(1-\frac{w_{k}(\mathbf{x})}{\omega(\mathbf{x})}\right) w_{k, l}\left(1-\frac{w_{l}(\mathbf{x})}{\omega(\mathbf{x})}\right) x_{l}$, in the diploid case we have

$$
\Delta \omega(\mathbf{x})=\omega\left(\mathbf{x}^{+}\right)-\omega(\mathbf{x})=R(\mathbf{x})+\frac{2}{\omega(\mathbf{x})}\left(\sum_{k} x_{k} w_{k}(\mathbf{x})^{2}-\omega(\mathbf{x})^{2}\right) \geq 0 .
$$

The mean fitness $\omega(\mathbf{x})=\mathbf{x}^{\prime} W \mathbf{x}$ for diploid populations, as a Lyapunov function, increases as time passes by, vanishing only when the process has reached equilibrium. Equation (10) constitutes the mean fitness increase theorem.

The partial rate of increase of the mean fitness due to frequency shifts only (see [8] $)$ is $\delta \omega(\mathbf{x}):=\sum_{k} \Delta x_{k} w_{k}(\mathbf{x})$. It satisfies

$$
\frac{\delta \omega(\mathbf{x})}{\omega(\mathbf{x})}=\sum_{k=1}^{K} x_{k}\left(\frac{w_{k}(\mathbf{x})}{\omega(\mathbf{x})}-1\right)^{2}=\sum_{k=1}^{K} \frac{\left(\Delta x_{k}\right)^{2}}{x_{k}}=\frac{1}{2} \bar{\sigma}_{A}^{2}(\mathbf{x})>0,
$$

where $\bar{\sigma}_{A}^{2}(\mathbf{x})$ is the allelic variance in relative fitness

$$
\bar{\sigma}_{A}^{2}(\mathbf{x}):=2 \sum_{k=1}^{K} x_{k}\left(\frac{w_{k}(\mathbf{x})}{\omega(\mathbf{x})}-1\right)^{2} .
$$

Equation (11) constitutes the diploid version of the Fisher fundamental theorem of natural selection under the Hardy-Weinberg condition involving random mating. See [28, [8] and [3].

2.1.4. Shahshahani gradient-like representation of the allelic dynamics (6). There is an alternative vectorial representation of the dynamics (6) emphasizing its gradient-like character. With $\mathbf{x} \in \mathcal{S}_{K}$, define the matrix $G(\mathbf{x})=D_{\mathbf{x}}-\mathbf{x x}^{\prime}$. It is symmetric, positive semi-definite whose quadratic form vanishes only for the constants and $G\left(\mathbf{e}_{k}\right)=0$ for all $k . G(\mathbf{x})$ is partially invertible on the space $\Delta_{K}$ orthogonal to the constants with left-inverse

$$
G(\mathbf{x})^{-1}=\left(I-\frac{1}{K} J\right) D_{\mathbf{x}}^{-1}
$$

so with $G(\mathbf{x})^{-1} G(\mathbf{x}) \boldsymbol{\delta}=\boldsymbol{\delta}$, for all $\boldsymbol{\delta} \in \Delta_{K}$, obeying $|\boldsymbol{\delta}|=0$. Note $G(\mathbf{x}) G(\mathbf{x})^{-1} \boldsymbol{\delta} \neq$ $\boldsymbol{\delta}$ and $|G(\mathbf{x}) \boldsymbol{\delta}|=0$ for all $\boldsymbol{\delta} \in \Delta_{K}$. Looking for a left-inverse in the weaker sense of the quadratic form, that is satisfying

$$
\boldsymbol{\delta}^{\prime} G(\mathbf{x})^{-1} G(\mathbf{x}) \boldsymbol{\delta}=\boldsymbol{\delta}^{\prime} I \boldsymbol{\delta}
$$

for all $\boldsymbol{\delta} \in \Delta_{K}$ with $|\boldsymbol{\delta}|=0$, every $G(\mathbf{x})^{-1}=\left(I-\frac{\lambda}{K} J\right) D_{\mathbf{x}}^{-1}$ would do for any real number $\lambda$. In particular $\lambda=0$, leading to $G(\mathbf{x})^{-1}=D_{\mathbf{x}}^{-1}$. 
Introduce the quantity $V_{W}(\mathbf{x})=\frac{1}{2} \log \omega(\mathbf{x})$. Then, (5) may be recast as the gradient-like dynamics:

$$
\Delta \mathbf{x}=\frac{1}{\omega(\mathbf{x})} G(\mathbf{x}) W \mathbf{x}=G(\mathbf{x}) \nabla V_{W}(\mathbf{x}),
$$

with $\Delta \mathbf{x} \in \Delta_{K}$, as a result of $|\Delta \mathbf{x}|=\mathbf{1}^{\prime} \Delta \mathbf{x}=0$ observing $\mathbf{1}^{\prime} G(\mathbf{x})=\mathbf{0}^{\prime}$. Note

$$
\nabla V_{W}(\mathbf{x})^{\prime} \Delta \mathbf{x}=\nabla V_{W}(\mathbf{x})^{\prime} G(\mathbf{x}) \nabla V_{W}(\mathbf{x}) \geq 0 .
$$

Based on [30, [31, the dynamics (13) is of gradient-type with respect to the Shahshahani-Svirezhev metric $G$. Its piecewise trajectories are perpendicular to the level surfaces of $V_{W}$ with respect to the scalar product given by

$$
\left\langle\boldsymbol{\delta}_{1}, \boldsymbol{\delta}_{2}\right\rangle_{G}=\left(\boldsymbol{\delta}_{1}^{\prime} G(\mathbf{x})^{-1} \boldsymbol{\delta}_{2}\right), \boldsymbol{\delta}_{1}, \boldsymbol{\delta}_{2} \in \Delta_{K} .
$$

We also have

$$
d_{G}\left(\mathbf{x}, \mathbf{x}^{\prime}\right)=\langle\Delta \mathbf{x}, \Delta \mathbf{x}\rangle_{G}^{1 / 2}=\left(\Delta \mathbf{x}^{\prime} G(\mathbf{x})^{-1} \Delta \mathbf{x}\right)^{1 / 2}=\left(\sum_{k=1}^{K} x_{k}^{-1}\left(\Delta x_{k}\right)^{2}\right)^{1 / 2} .
$$

From (11) and (12), $d_{G}\left(\mathbf{x}, \mathbf{x}^{\prime}\right)$, which is the length of $\Delta \mathbf{x}$, is also the square-root of half the allelic variance (the standard deviation) in relative fitness.

2.2. Frequency-dependent fitness. Consider the dynamics (6) in vector form. Let the frequency-dependent marginal fitness of $A_{k}$ be defined by $w_{k}(\mathbf{x})>0, k=$ $1, \ldots, K$, not necessarily of the linear form $(W \mathbf{x})_{k}$ for some fitness matrix $W$. With $\mathbf{w}(\mathbf{x}):=\left(w_{1}(\mathbf{x}), \ldots, w_{K}(\mathbf{x})\right)^{\prime}$ a new column vector of frequency-dependent marginal fitnesses, we can first think of defining the dynamics on $\mathcal{S}_{K}$ by

$$
\mathbf{x}^{+}=\frac{1}{\omega(\mathbf{x})} D_{\mathbf{x}} \mathbf{w}(\mathbf{x})=: \mathbf{p}(\mathbf{x}),
$$

where $\omega(\mathbf{x}):=\mathbf{x}^{\prime} \mathbf{w}(\mathbf{x})$ is the new mean fitness of the allelic population. Unless $\mathbf{w}(\mathbf{x})=W \mathbf{x}$ as before, such dynamics cannot be of Shahshahani-gradient type. This suggests to consider the alternative gradient-like dynamics to (14), still on the simplex:

$$
\mathbf{x}^{+}=\mathbf{x}+G(\mathbf{x}) \nabla V_{W}(\mathbf{x})=: \mathbf{p}(\mathbf{x})
$$

where $V_{W}(\mathbf{x})=\frac{1}{2} \log \omega(\mathbf{x})$ and $\omega(\mathbf{x}):=\mathbf{x}^{\prime} \mathbf{w}(\mathbf{x})=\sum_{k} x_{k} w_{k}(\mathbf{x})$. A particular case is $\mathbf{w}(\mathbf{x})=W(\mathbf{x}) \mathbf{x}$ where $W$ is now frequency-dependent and symmetric for each $\mathbf{x}$ and $\omega(\mathbf{x})=\mathbf{x}^{\prime} W(\mathbf{x}) \mathbf{x}$. Fitness landscapes can be more general than quadratic forms.

Examples:

- Suppose $W(\mathbf{x})_{k, l}=\sum_{j=1}^{K} W_{k, l}^{j} x_{j}$ where $W_{k, l}^{j}=W_{l, k}^{j}$ for all $j, k, l \in\{1, \ldots, K\}$. Then the mean fitness $\omega(\mathbf{x})=\mathbf{x}^{\prime} W(\mathbf{x}) \mathbf{x}$ is homogeneous of degree $d=3$ in the variables $\mathbf{x}$.

- With $\sigma, q>0$, let $\boldsymbol{\sigma}_{q}(\mathbf{x})=\sigma\left(x_{1}^{q-2}, \ldots, x_{K}^{q-2}\right)^{\prime}$ and suppose $W(\mathbf{x})=D_{\boldsymbol{\sigma}_{q}(\mathbf{x})}$ is diagonal, so that $\omega(\mathbf{x})=\sigma \sum_{k=1}^{K} x_{k}^{q}$. Such selection models were considered in 34, [10, 13, 17] and [4, in relation to heterozygozity. $\diamond$ 


\section{Evolution DRIVEn By MUtation}

We now briefly describe the frequency distribution dynamics when mutation is the only driving source of motion.

Assume alleles mutate according to the scheme: $A_{k} \rightarrow A_{l}$ with probability $\mu_{k, l} \in$ $[0,1]$ satisfying $\mu_{k, k}=0$ and $0<\sum_{l \neq k} \mu_{k, l} \leq 1$ for all $k$. Let $M:=\left[\mu_{k, l}\right]$ be the mutation pattern matrix; we shall assume that the non-negative matrix $M$ is irreducible.

3.1. Frequency dynamics under mutation only. Considering first an updating mechanism of the frequencies where only mutations operate, we get

$$
x_{k}^{+}=x_{k}+\sum_{l \neq k} \mu_{l, k} x_{l}-x_{k} \sum_{l \neq k} \mu_{k, l}, k=1, \ldots, K,
$$

whose meaning is the one of a "master equation". In vector form, with $M^{\prime}$ the transpose of $M$

$$
\mathbf{x}^{+}=\mathbf{x}+M^{\prime} \mathbf{x}-D_{M \mathbf{1}} \mathbf{x}=: \mathbf{M x}=: \mathbf{p}_{M}(\mathbf{x}),
$$

and the update of the frequencies with mutations is given by the linear transformation

$$
\mathbf{M}:=I-D_{M 1}+M^{\prime} .
$$

The vector $\mathbf{m}:=M \mathbf{1}$ is called the mutation load. We have $\mathbf{M} \geq \mathbf{0}$ and $\mathbf{M}=M^{\prime}$ if and only if $M$ is stochastic: $\mathbf{m}=M \mathbf{1}=\mathbf{1}$,meaning

$$
\sum_{l \neq k} \mu_{k, l}=1 \text { for all } k \text {. }
$$

Also $\mathbf{1}^{\prime} \mathbf{M}=\mathbf{1}^{\prime}$ and then $\mathbf{M}$ maps $\mathcal{S}_{K}$ into $\mathcal{S}_{K}$ because if $\mathbf{1}^{\prime} \mathbf{x}=1$, then $\mathbf{1}^{\prime} \mathbf{x}^{+}=$ $\mathbf{1}^{\prime} \mathbf{M x}=\left(\mathbf{M}^{\prime} \mathbf{1}\right)^{\prime} \mathbf{x}=\mathbf{1}^{\prime} \mathbf{x}=1$. The matrix $\mathbf{M}^{\prime}$ is stochastic and irreducible and so, by Perron-Frobenius theorem, it has a unique strictly positive probability lefteigenvector associated to the real dominant eigenvalue 1. Let $\mathbf{x}_{e q}^{\prime}$ be this row-vector, so obeying $\mathbf{x}_{e q}^{\prime}=\mathbf{x}_{e q}^{\prime} \mathbf{M}^{\prime}$, or $\mathbf{x}_{e q}=\mathbf{M} \mathbf{x}_{e q}$. Under the irreducibility assumption on $M$, the frequencies dynamics involving only mutations has a unique polymorphic equilibrium fixed point $\mathbf{x}_{e q}>\mathbf{0}$. When $\mathbf{M}$ is primitive then $\lim _{r \rightarrow \infty} \mathbf{M}^{r}=\mathbf{x}_{e q} \mathbf{1}^{\prime}$. This shows that, at generation $r$,

$$
\mathbf{x}(r)=\mathbf{M}^{r} \mathbf{x}(0) \underset{r \rightarrow \infty}{\rightarrow} \mathbf{x}_{e q} \mathbf{1}^{\prime} \mathbf{x}(0)=\mathbf{x}_{e q},
$$

regardless of the initial condition $\mathbf{x}(0)$ belonging to $\mathcal{S}_{K}$. The equilibrium vector $\mathbf{x}_{e q}$ is asymptotically stable. These considerations are the same as limit (ergodic) theorems for Markov chains.

Note finally that from (17):

$$
\Delta \mathbf{x}=(\mathbf{M}-I) \mathbf{x}=: \nabla \mathcal{V}_{M}(\mathbf{x}),
$$

where $\mathcal{V}_{M}(\mathbf{x})=\frac{1}{2} \mathbf{x}^{\prime}(\mathbf{M}-I) \mathbf{x}$ is the quadratic mutation potential. The probability right-eigenvector $\mathbf{x}_{e q}$ of $\mathbf{M}$ uniquely solves $\nabla \mathcal{V}_{M}(\mathbf{x})=0$ with $\mathcal{V}_{M}\left(\mathbf{x}_{e q}\right)=0$, maximal. 
3.2. Special mutation patterns. There are many special mutation scenarii which deserve interest.

(i) Reversible mutations: Let $\mathbf{x}_{e q}$ solve $\mathbf{x}_{e q}^{\prime}=\mathbf{x}_{e q}^{\prime} \mathbf{M}^{\prime}$. Define

$$
\overleftarrow{\mathbf{M}^{\prime}}=D_{\mathbf{x}_{e q}}^{-1} \mathbf{M} D_{\mathbf{x}_{e q}}
$$

We have $\overleftarrow{\mathbf{M}^{\prime}} \mathbf{1}=D_{\mathbf{x}_{e q}}^{-1} \mathbf{M} \mathbf{x}_{e q}=\mathbf{1}$, so $\overleftarrow{\mathbf{M}^{\prime}}$ is the stochastic matrix of the time-reversed mutation process at equilibrium with invariant measure $\mathbf{x}_{e q}^{\prime}>\mathbf{0}^{\prime}$. If $\overleftarrow{\mathbf{M}^{\prime}}=\mathbf{M}^{\prime}$, then the mutation pattern is said to be time-reversible (detailed balance holds). In this case

$$
\mu_{l, k}=\mu_{k, l} \frac{x_{e q, k}}{x_{e q, l}}
$$

with $\mu_{k, l}>0 \Rightarrow \mu_{l, k}>0$. When reversible, the matrix $\mathbf{M}^{\prime}$ is diagonally similar to the matrix $\mathbf{M}_{S}^{\prime}:=D_{\mathbf{x}_{e q}}^{1 / 2} \mathbf{M}^{\prime} D_{\mathbf{x}_{e q}}^{-1 / 2}$ which is symmetric with real eigenvalues, so $\mathbf{M}^{\prime}$ and $\mathbf{M}$ have real eigenvalues.

(ii) If $M=M^{\prime}$, then $\mathbf{M}=\mathbf{M}^{\prime}$ and $\mathbf{M}$ is symmetric itself and thus doubly stochastic. In that case, $\mathbf{x}_{e q}=\frac{1}{K} \cdot(1, \ldots, 1)^{\prime}=: \mathbf{x}_{b}$ (the barycenter of $\mathcal{S}_{K}$ ) and such mutation patterns are reversible. Let us give some Examples:

- A model with symmetric mutations is obtained for instance while assuming multiplicative mutations: $\mu_{k, l}=\mu_{k} \mu_{l}$. In this case, with $\boldsymbol{\mu}$ the column vector of the $\mu_{k} \mathrm{~s}, k=1, \ldots, K$,

$$
\mathbf{M}=I+\boldsymbol{\mu} \boldsymbol{\mu}^{\prime}-|\boldsymbol{\mu}| D_{\boldsymbol{\mu}} .
$$

This mutation pattern is reversible with $\mathbf{x}_{e q}=\mathbf{x}_{b}$.

- Alternatively, assuming $\mu_{k, l} \equiv \mu \in\left(0, \frac{1}{K-1}\right]$ for all $k \neq l$ leads to $\mathbf{M}=\mu J+$ $(1-K \mu) I$ which is also symmetric.

- Alternatively, while considering additive mutations: $\mu_{k, l}=\left(\mu_{k}+\mu_{l}\right) / 2$, with $\boldsymbol{\mu}^{\prime}=\left(\mu_{1}, \ldots, \mu_{K}\right)$,

$$
\mathbf{M}=\left(1-\frac{1}{2}|\boldsymbol{\mu}|\right) I-\frac{K}{2} D_{\boldsymbol{\mu}}+\frac{1}{2}\left(\boldsymbol{\mu \mathbf { 1 } ^ { \prime } + \mathbf { 1 } \boldsymbol { \mu } ^ { \prime } )}\right.
$$

and $\mathbf{x}_{e q}=\mathbf{x}_{b}$. This mutation pattern is reversible.

(iii) It is not necessary that $M=M^{\prime}$ in order to have $\mathbf{M}$ doubly stochastic. It suffices to impose $M \mathbf{1}=M^{\prime} \mathbf{1}$. In that case although $\mathbf{M} \neq \mathbf{M}^{\prime}$, the overall inputoutput mutation probabilities attached to any state coincide and the equilibrium state again matches with the barycenter $\mathbf{x}_{b}$ of $\mathcal{S}_{K}$. But since $M \neq M^{\prime}$, such mutation patterns are not reversible.

(iv) Assume the mutation probabilities only depend on the initial state, that is: $\mu_{k, l}=\mu_{k}$ for all $l \neq k$. Then

$$
\mathbf{M}=I-K D_{\mu}+\mathbf{1} \boldsymbol{\mu}^{\prime}
$$


This mutation model is not reversible because

$$
\overleftarrow{\mathbf{M}^{\prime}}=D_{\boldsymbol{\mu}}^{-1} \mathbf{M} D_{\boldsymbol{\mu}}=I-K D_{\boldsymbol{\mu}}+D_{\boldsymbol{\mu}}^{-1} \mathbf{1} \boldsymbol{\mu}^{\prime} D_{\boldsymbol{\mu}} \neq \mathbf{M}^{\prime}=I-K D_{\boldsymbol{\mu}}+\boldsymbol{\mu} \mathbf{1}^{\prime}
$$

If $\mu_{k}>0$ for all $k$, the equilibrium state is

$$
x_{e q, k}=\frac{1 / \mu_{k}}{\sum_{l} 1 / \mu_{l}} \text { or } \mathbf{x}_{e q}=\frac{1}{\operatorname{trace}\left(D_{\boldsymbol{\mu}}^{-1}\right)} D_{\boldsymbol{\mu}}^{-1} \mathbf{1} .
$$

$(v)$ One-way (irreversible) mutations: assume $\mu_{k, l}>0 \Rightarrow \mu_{l, k}=0$. This model is clearly not reversible and, when the associated mutation matrix $M$ is irreducible, it has a non-trivial $\mathbf{x}_{e q}>\mathbf{0}$. This model includes the cyclic mutation pattern for which $\mu_{k, l}=\mu_{k} \delta_{l, k+1}, k=1, \ldots, K-1$ and $\mu_{K, l}=\mu_{K} \delta_{l, 1}$, with

$$
\mathbf{x}_{e q}=\frac{1}{\operatorname{trace}\left(D_{\boldsymbol{\mu}}^{-1}\right)} D_{\boldsymbol{\mu}}^{-1} \mathbf{1}
$$

as in the previous example $(i v)$.

(vi) (Kingman house-of-cards mutations, 22]).

Assume the mutation probabilities now only depend on the terminal state, that is: $\mu_{k, l}=\mu_{l}$ for all $k \neq l$, still with $\mu_{k, k}=0$. Throughout, we assume $\mu_{k}>0$. Let $\boldsymbol{\mu}^{\prime}=\left(\mu_{1}, \ldots, \mu_{K}\right)$. Then, $M=\mathbf{1} \boldsymbol{\mu}^{\prime}-D_{\boldsymbol{\mu}}, M \mathbf{1}=|\boldsymbol{\mu}| \cdot \mathbf{1}-\boldsymbol{\mu}$ where $\min \mu_{k}<|\boldsymbol{\mu}|:=$ $\boldsymbol{\mu}^{\prime} \mathbf{1}<1+\max \mu_{k}, \mathbf{M}=\boldsymbol{\mu} \mathbf{1}^{\prime}+(1-|\boldsymbol{\mu}|) I$ and

$$
\mathbf{x}^{+}=\mathbf{M x}=\mathbf{x}+M^{\prime} \mathbf{x}-D_{\mathbf{x}} M \mathbf{1}=\boldsymbol{\mu}+(1-|\boldsymbol{\mu}|) \mathbf{x} .
$$

The equilibrium state is $\mathbf{x}_{e q}=\boldsymbol{\mu} /|\boldsymbol{\mu}|$ and it is stable. Note that $|\boldsymbol{\mu}| \leq 1+\frac{1}{K-1}$. This mutation model is reversible because

$$
\overleftarrow{\mathbf{M}^{\prime}}=D_{\boldsymbol{\mu}}^{-1} \mathbf{M} D_{\boldsymbol{\mu}}=D_{\boldsymbol{\mu}}^{-1} \boldsymbol{\mu} \mathbf{1}^{\prime} D_{\boldsymbol{\mu}}+(1-|\boldsymbol{\mu}|) I=\mathbf{1} \boldsymbol{\mu}^{\prime}+(1-|\boldsymbol{\mu}|) I=\mathbf{M}^{\prime}
$$

In this model the coordinates are decoupled: $x_{k}^{+}=\mu_{k}+(1-|\boldsymbol{\mu}|) x_{k}$, depends only on $x_{k}$. We shall come back at length to this mutation pattern in the sequel.

- Note that if $\mathbf{m}=M \mathbf{1}=\mathbf{1}$, namely if $(|\boldsymbol{\mu}|-1) \cdot \mathbf{1}=\boldsymbol{\mu}$, then $\mathbf{x}_{e q}=\mathbf{x}_{b}, K(|\boldsymbol{\mu}|-1)=$ $|\boldsymbol{\mu}|($ else $|\boldsymbol{\mu}|-1=1 /(K-1))$ and

$$
\mathbf{x}^{+}=\boldsymbol{\mu}+(1-|\boldsymbol{\mu}|) \mathbf{x}=(|\boldsymbol{\mu}|-1)(\mathbf{1}-\mathbf{x})=\frac{1}{K-1}(\mathbf{1}-\mathbf{x})
$$

- In the latter case, $|\boldsymbol{\mu}|>1$ in particular. If $|\boldsymbol{\mu}|>1$ and $\mathbf{m} \neq \mathbf{1}$, in view of $\Delta \mathbf{x}=|\boldsymbol{\mu}|\left(\mathbf{x}_{e q}-\mathbf{x}\right), \mathbf{x}$ goes fast to $\mathbf{x}_{e q}$.

- Another very special case is when $|\boldsymbol{\mu}|=1$. Here $\mathbf{M}=\boldsymbol{\mu} \mathbf{1}^{\prime}$ and $\mathbf{x}^{+}=\mathbf{M x}=\boldsymbol{\mu}$. Starting from any initial condition, the dynamics moves in one-step to $\mathbf{x}_{e q}=\boldsymbol{\mu}$ (inside the simplex $\mathcal{S}_{K}$ ) and stays there for ever. $\diamond$

\section{Evolution DRIVEn By COMBINED Fitness AND MUTATION FORCES}

Let us now consider the dynamics driven both by fitness and mutation. 
4.1. The combined fitness/mutation frequency dynamics; [15], 26], 14, 20]. Combining the fitness and mutation effects consists in applying first the fitness mapping and then let mutation act on the result. Proceeding in this way, we get the 'fitness-first' dynamics [15]:

$$
\mathbf{x}^{+}=\frac{1}{\mathbf{x}^{\prime} W \mathbf{x}} \mathbf{M} D_{W \mathbf{x}} \mathbf{x}=\frac{1}{\mathbf{x}^{\prime} W \mathbf{x}} \mathbf{M} D_{\mathbf{x}} W \mathbf{x},
$$

defining a new nonlinear transformation. Alternatively, $\mathbf{x}^{+}=\mathbf{p}(\mathbf{x})$ where $\mathbf{p}(\mathbf{x})=$ $\frac{1}{\mathbf{x}^{\prime} W \mathbf{x}} \mathbf{M} D_{\mathbf{x}} W \mathbf{x}$ is the new mapping from $\mathcal{S}_{K}$ to $\mathcal{S}_{K}$ to consider. Component-wise, this is also as required

$$
x_{k}^{+}=\frac{1}{\omega(\mathbf{x})}\left(x_{k} w_{k}(\mathbf{x})+\sum_{l \neq k} \mu_{l, k} w_{l}(\mathbf{x}) x_{l}-x_{k} w_{k}(\mathbf{x}) \sum_{l \neq k} \mu_{k, l}\right), k=1, \ldots, K .
$$

We have: $\mathbf{p}\left(\mathbf{e}_{k}\right)=\left(\mu_{k, 1}, \ldots, \mu_{k, k-1}, 1-\sum_{l \neq k} \mu_{k, l}, \mu_{k, k+1}, \ldots, \mu_{k, K}\right)^{\prime} \in \mathcal{S}_{K}$ and so the extremal states $\mathbf{e}_{k}$ are not invariant under $\mathbf{p}$ and from the fixed-point theorem, there exists some equilibrium state in $\mathcal{S}_{K}$. Using the representation (13), (17) and (18):

$$
\Delta \mathbf{x}=(\mathbf{M}-I) \mathbf{x}+\mathbf{M} G(\mathbf{x}) \nabla V_{W}(\mathbf{x})=\nabla \mathcal{V}_{M}(\mathbf{x})+\mathbf{M} G(\mathbf{x}) \nabla V_{W}(\mathbf{x}) .
$$

This is not a Shahshahani gradient-like dynamics in general. 15 also considers a continuous-time version of (22).

When $\mathbf{M}=I$ (no mutation) (22) boils down into (13) and when $W=J$ (no selection), (22) boils down into (18).

When both $\mathbf{M}=I$ (no mutation) and $W=J$ (no selection), $\Delta \mathbf{x}=0$ with corresponding neutral $\mathbf{p}(\mathbf{x})=\mathbf{x}$.

4.2. Fitness/mutation frequency dynamics in the house of cards condition for mutations. This is a remarkable case where the allelic dynamics driven both by fitness and mutation forces has a Shahshahani gradient-like structure, 15. Indeed, from (19), $\mathbf{M}=\boldsymbol{\mu} \mathbf{1}^{\prime}+(1-|\boldsymbol{\mu}|) I$ and (20) boils down to

$$
\mathbf{x}^{+}=\boldsymbol{\mu}+(1-|\boldsymbol{\mu}|) \frac{1}{\mathbf{x}^{\prime} W \mathbf{x}} D_{W \mathbf{x}} \mathbf{x} .
$$

We now have

$$
(\mathbf{M}-I) \mathbf{x}=\boldsymbol{\mu}-|\boldsymbol{\mu}| \mathbf{x}=G(\mathbf{x}) \nabla V_{M}(\mathbf{x}),
$$

where $V_{M}(\mathbf{x})=\sum_{k} \mu_{k} \log x_{k}-|\boldsymbol{\mu}| \sum_{k} x_{k}$. Indeed, $\nabla V_{M}(\mathbf{x})=D_{\mathbf{x}}^{-1} \boldsymbol{\mu}-|\boldsymbol{\mu}| \mathbf{1}$ and

$$
\left(D_{\mathbf{x}}-\mathbf{x x}^{\prime}\right) \nabla V_{M}(\mathbf{x})=\boldsymbol{\mu}-|\boldsymbol{\mu}| \mathbf{x} .
$$

Furthermore,

$$
\mathbf{M} G(\mathbf{x})=\left(\boldsymbol{\mu} \mathbf{1}^{\prime}+(1-|\boldsymbol{\mu}|) I\right)\left(D_{\mathbf{x}}-\mathbf{x} \mathbf{x}^{\prime}\right)=(1-|\boldsymbol{\mu}|)\left(D_{\mathbf{x}}-\mathbf{x} \mathbf{x}^{\prime}\right) .
$$

Thus (22) can alternatively be written as

$$
\Delta \mathbf{x}=G(\mathbf{x})\left(\nabla V_{M}(\mathbf{x})+(1-|\boldsymbol{\mu}|) \nabla V_{W}(\mathbf{x})\right)=: G(\mathbf{x}) \nabla V(\mathbf{x})
$$


which is of Shahshahani gradient-type with combined mutation/selection potential

$$
\begin{aligned}
& V(\mathbf{x})=V_{M}(\mathbf{x})+(1-|\boldsymbol{\mu}|) V_{W}(\mathbf{x}) \\
& =\sum_{k} \mu_{k} \log x_{k}-|\boldsymbol{\mu}|+\frac{1}{2}(1-|\boldsymbol{\mu}|) \log \mathbf{x}^{\prime} W \mathbf{x} \\
& =: \log \mathcal{W}(\mathbf{x}), \text { where } \mathcal{W}(\mathbf{x})=e^{-|\boldsymbol{\mu}|} \prod_{k=1}^{K} x_{k}^{\mu_{k}}\left(\mathbf{x}^{\prime} W \mathbf{x}\right)^{(1-|\boldsymbol{\mu}|) / 2} .
\end{aligned}
$$

Note that $\mathcal{W}(\mathbf{x})$ is homogeneous of degree 1 .

4.3. House of cards and polymorphism. If some polymorphic state $\mathbf{x}_{e q}$ exists in the interior of the simplex, then $\mathbf{x}_{e q}=\mathbf{z} /|\mathbf{z}|$ where $\mathbf{z}>\mathbf{0}$ solves

$$
\begin{aligned}
\nabla V(\mathbf{z}) & =D_{\mathbf{z}}^{-1} \boldsymbol{\mu}+(1-|\boldsymbol{\mu}|) \frac{W \mathbf{z}}{\mathbf{z}^{\prime} W \mathbf{z}}=\lambda \mathbf{1}, \text { else } \\
\lambda \mathbf{z} & =\boldsymbol{\mu}+(1-|\boldsymbol{\mu}|) \frac{D_{\mathbf{z}} W \mathbf{z}}{\mathbf{z}^{\prime} W \mathbf{z}}
\end{aligned}
$$

for some arbitrary Lagrangian parameter $\lambda(\mathbf{z}$ is an extremum of $V$ under the constraint $|\mathbf{z}|$ fixed).

- If $\boldsymbol{\mu}=\mathbf{0}$ (no mutation), the searched $\mathbf{z}=\mathbf{z}_{S}>\mathbf{0}$ is the one solving, as required, $W \mathbf{z}_{S}=\boldsymbol{\lambda}\left(\mathbf{z}_{S}^{\prime} W \mathbf{z}_{S}\right) \mathbf{1}$ up to a multiplicative constant, else $W \mathbf{z}_{S}=\mathbf{1}$ if $\lambda=1 /\left|\mathbf{z}_{S}\right|$.

- If $W=J$ (no selection), $\frac{D_{\mathbf{z}} W \mathbf{z}}{\mathbf{z}^{\prime} W \mathbf{z}}=\frac{\mathbf{z}}{|\mathbf{z}|}$ and the searched $\mathbf{z}=\mathbf{z}_{M}>\mathbf{0}$ is the one solving, as required, $\lambda \mathbf{z}_{M}=\boldsymbol{\mu}+(\mathbf{1}-|\boldsymbol{\mu}|) \mathbf{z}_{M} /\left|\mathbf{z}_{M}\right|$ up to a multiplicative constant so that $\mathbf{z}_{M}=\boldsymbol{\mu}$ if $\lambda=1+(1-|\boldsymbol{\mu}|) /\left|\mathbf{z}_{M}\right|$.

So $\mathbf{x}_{e q}=\mathbf{z} /|\mathbf{z}|$ where $\mathbf{z}>\mathbf{0}$ is some fixed point of the map

$$
\mathbf{x} \rightarrow T_{\boldsymbol{\mu}}(\mathbf{x})=\lambda^{-1}\left(\boldsymbol{\mu}+(1-|\boldsymbol{\mu}|) \frac{D_{\mathbf{x}} W \mathbf{x}}{\mathbf{x}^{\prime} W \mathbf{x}}\right) .
$$

If there is a stable fixed point $\mathbf{z}_{S}>0$ of $T_{\mathbf{0}}$, such that $W \mathbf{z}_{S}=\mathbf{1}$ (the purely selection dynamics has a stable polymorphic equilibrium state $\mathbf{z}_{S} /\left|\mathbf{z}_{S}\right|$ in the simplex), then clearly, as long as $|\boldsymbol{\mu}| \leq 1, \mathbf{z}>\mathbf{0}$, as a fixed point of $T_{\boldsymbol{\mu}}$, exists as well and is unique, as a maximum of the concave potential $V(\mathbf{x})$. If $\mathbf{z}_{S} /\left|\mathbf{z}_{S}\right|$ is stable indeed, then so is $\mathbf{x}_{e q}=\mathbf{z} /|\mathbf{z}|$ if $|\boldsymbol{\mu}| \leq 1$, because the new mean fitness function $w_{\boldsymbol{\mu}}(\mathbf{x}):=\mathcal{W}(\mathbf{x})$ inherits the concavity of $\mathbf{x}^{\prime} W \mathbf{x}=: w_{\mathbf{0}}(\mathbf{x})=w(\mathbf{x})$ if $|\boldsymbol{\mu}|<1$ and is concave as well if $|\boldsymbol{\mu}|=1$. If $|\boldsymbol{\mu}|=1$, then $\mathbf{x}_{e q}=\boldsymbol{\mu} /|\boldsymbol{\mu}|$. Note however that $|\boldsymbol{\mu}|>1$ entails that a stable $\mathbf{z}_{S} /\left|\mathbf{z}_{S}\right|$ can be switched to an unstable $\mathbf{z} /|\mathbf{z}|$ : strong mutations $(|\boldsymbol{\mu}|>1)$ can destroy a stable polymorphic state of the selection equation.

\section{Evolutionary Dynamics: the Stochastic POINT OF VIEW}

We now consider a stochastic version of evolutionary dynamics biased by selection/mutation effects, thereby adding a "random genetic drift" to the deterministic dynamics.

5.1. Random genetic drift and the Wright-Fisher model. We will consider the multi-allelic Wright-Fisher model with bias, (see [8], section 5.10). 
5.1.1. The discrete-time model and first properties. Consider a multi-allelic population with constant size $N$. In the haploid (diploid) case, $N$ is (twice) the number $N_{e}$ of effective loci. Let $\mathbf{n}:=\left(n_{k} ; k=1, \ldots, K\right)$ and $\mathbf{n}^{+}:=\left(n_{k}^{+} ; k=1, \ldots, K\right)$ be two vectors of integers quantifying the size of the allelic populations at two consecutive generations $r \varepsilon$ and $r \varepsilon+\varepsilon$, where $\varepsilon>0$ is some small parameter fixing the time elapsed between two consecutive generations. With $|\mathbf{n}|=\sum_{k} n_{k}=N$, therefore $\left|\frac{\mathbf{n}}{N}\right|=\left|\frac{\mathbf{n}^{\prime}}{N}\right|=1$ and both $\frac{\mathbf{n}}{N}$ and $\frac{\mathbf{n}^{\prime}}{N}$ belong to $\mathcal{S}_{K}$. Suppose the stochastic evolutionary dynamics is now given by a Markov chain whose one-step probability transition matrix $P$ from state $\mathbf{N}=\mathbf{n}$ to state $\mathbf{N}^{+}=\mathbf{n}^{+}$is given by the multinomial Wright-Fisher model

$$
\mathbf{P}\left(\mathbf{N}_{(r+1) \varepsilon}=\mathbf{n}^{+} \mid \mathbf{N}_{r \varepsilon}=\mathbf{n}\right)=: P\left(\mathbf{n}, \mathbf{n}^{+}\right)=\left(\begin{array}{c}
N \\
n_{1}^{+} \cdots n_{K}^{+}
\end{array}\right) \prod_{k=1}^{K} p_{k}\left(\frac{\mathbf{n}}{N}\right)^{n_{k}^{+}} .
$$

Here the $p_{k}$ s are the coordinates of some mapping $\mathbf{p}: \mathcal{S}_{K} \rightarrow \mathcal{S}_{K}$, translating some bias from neutrality (where $\mathbf{p}$ is simply the identity). The state-space dimension of this Markov chain is $\left(\begin{array}{c}N+K-1 \\ K-1\end{array}\right)$ (the number of compositions of integer $N$ into $K$ non-negative parts). In other words, with $\mathbf{x}:=\frac{\mathbf{n}}{N} \in \mathcal{S}_{K}$,

$$
\left(\mathbf{N}_{(r+1) \varepsilon}=\mathbf{n}^{\prime} \mid \mathbf{N}_{r \varepsilon}=\mathbf{n}\right) \sim \operatorname{multinomial}(N, \mathbf{p}(\mathbf{x})) .
$$

5.1.2. Scalings. If we assume that $\mathbf{p}(\mathbf{x})-\mathbf{x}=\varepsilon \mathbf{f}(\mathbf{x})$ for some drift function $\mathbf{f}(\mathbf{x})$ (meaning $\Delta \mathbf{x}=\varepsilon \mathbf{f}(\mathbf{x})$ is the frequency shift per generation of duration $\Delta t=\varepsilon$ ), from the mean and covariance structure of multinomial distributions, with $t=r \varepsilon$, $\Delta \mathbf{N}_{t}=\left(\mathbf{N}_{t+\varepsilon}-\mathbf{N}_{t}\right)$, we get

$$
\begin{aligned}
& \mathbf{E}_{\mathbf{x}}\left(\Delta \mathbf{N}_{t}\right)=\varepsilon N \mathbf{f}(\mathbf{x}) \\
& \sigma_{\mathbf{x}}^{2}\left(\Delta \mathbf{N}_{t}\right)=\varepsilon N\left(D_{\mathbf{p}(\mathbf{x})}-\mathbf{p}(\mathbf{x}) \mathbf{p}(\mathbf{x})^{\prime}\right) .
\end{aligned}
$$

With $\mathbf{x}_{t}:=\mathbf{N}_{t} / N$ and $\Delta \mathbf{x}_{t}:=\mathbf{x}_{t+\varepsilon}-\mathbf{x}_{t}=\left(\mathbf{N}_{t+\varepsilon}-\mathbf{N}_{t}\right) / N$, to the first-order in $\varepsilon$,

$$
\begin{aligned}
\mathbf{E}_{\mathbf{x}}\left(\Delta \mathbf{x}_{t}\right) & =\varepsilon \mathbf{f}(\mathbf{x}) \\
\sigma_{\mathbf{x}}^{2}\left(\Delta \mathbf{x}_{t}\right) & =\varepsilon \frac{N}{N^{2}}\left(D_{\mathbf{p}(\mathbf{x})}-\mathbf{p}(\mathbf{x}) \mathbf{p}(\mathbf{x})^{\prime}\right)=\frac{\varepsilon}{N}\left(D_{\mathbf{x}}-\mathbf{x} \mathbf{x}^{\prime}\right)+o(\varepsilon) \sim \frac{\varepsilon}{N} G(\mathbf{x}),
\end{aligned}
$$

since the deviation of $\mathbf{p}(\mathbf{x})$ from $\mathbf{x}$ induce second-order effects in $\varepsilon$ on the variance. This means

$$
\Delta \mathbf{x}_{t}=\mathbf{f}\left(\mathbf{x}_{t}\right) \varepsilon+\sqrt{\varepsilon} \frac{1}{\sqrt{N}} G^{1 / 2}\left(\mathbf{x}_{t}\right) \boldsymbol{\xi}_{t+\varepsilon}
$$

where $\left(\boldsymbol{\xi}_{t} ; t \in\{\varepsilon, 2 \varepsilon, \ldots\}\right)$ is an iid $K$-dimensional Gaussian sequence with zero mean and covariance matrix $I$. For any $\delta>0$, using the multinomial structure of the Wright-Fisher transition matrix, we have

$$
\lim _{\varepsilon \rightarrow 0} \frac{1}{\varepsilon} \int_{|\mathbf{y}-\mathbf{x}|>\delta} \mathbf{P}\left(\mathbf{x}_{t+\varepsilon} \in d \mathbf{y} \mid \mathbf{x}_{t}=\mathbf{x}\right)=0 .
$$

The stochastic dynamics (26) is a discretized version of the diffusion process with continuous sample paths on $\mathcal{S}_{K}$ (making $\varepsilon \rightarrow 0$ )

$$
d \mathbf{x}_{t}=\mathbf{f}\left(\mathbf{x}_{t}\right) d t+\frac{1}{\sqrt{N}} G^{1 / 2}\left(\mathbf{x}_{t}\right) d \mathbf{w}_{t}
$$


where $\mathbf{w}_{t}$ is a $K$-dimensional standard Brownian motion. Such results on the diffusive approximation could be made rigorous using theorems from [6].

5.1.3. Speed (invariant) densities of the scaled process. The speed density of the diffusion process (27), cancelling the probability flux of its Kolmogorov forward (or Fokker-Planck) equation, is (up to a multiplicative constant)

$$
m_{N}(\mathbf{x})=\operatorname{det}\left(G^{-1}(\mathbf{x})\right) \exp 2 N \int^{\mathbf{x}}\left(G^{-1} \mathbf{f}\right)(\mathbf{y}) \cdot d \mathbf{y},
$$

possibly not normalizable into a probability density function. If, for some potential $V, \mathbf{f}(\mathbf{x})=G(\mathbf{x}) \nabla V(\mathbf{x})$, then, by applying the gradient theorem to the line integral $\int^{\mathbf{x}}\left(G^{-1} \mathbf{f}\right)(\mathbf{y}) \cdot d \mathbf{y}$ over some path in $\mathcal{S}_{K}$ ending in $\mathbf{x}$

$$
m_{N}(\mathbf{x})=\prod_{k=1}^{K} x_{k}^{-1} \exp 2 N V(\mathbf{x}) .
$$

It may happen that, for some suitable choice of $V$, some normalizing constant $Z_{N}$ turns $m_{N}$ to a probability density $p_{N}(\mathbf{x})=Z_{N}^{-1} m_{N}(\mathbf{x})$. In such cases, $p_{N}(\mathbf{x})$ is the limiting equilibrium probability distribution of some random variable $\mathbf{X}_{N} \sim$ $p_{N}(\mathbf{x})$ describing the asymptotic allelic frequencies. Note that a non-integrable case is the neutral case with no $\operatorname{drift} \mathbf{f}(\mathbf{x}) \equiv \mathbf{0}$ (or $\mathbf{p}(\mathbf{x})=\mathbf{x}$ ), with speed measure $m_{N}(\mathbf{x})=\prod_{k=1}^{K} x_{k}^{-1}$ on the simplex, but also $\mathbf{f}(\mathbf{x})=G(\mathbf{x}) \nabla V_{W}(\mathbf{x})$ as in (13), with $V_{W}(\mathbf{x})=\frac{1}{2} \log \mathbf{x}^{\prime} W \mathbf{x}$, and non-summable associated speed measure $m_{N}(\mathbf{x})=$ $\left(\prod_{k=1}^{K} x_{k}^{-1}\right)\left(\mathbf{x}^{\prime} W \mathbf{x}\right)^{N}$. We shall now consider situations including mutations where $m_{N}(\mathbf{x})$ is integrable and thus normalizable.

5.1.4. The mutation/selection potential under the house of cards condition for mutationd. For instance, if now $V(\mathbf{x})$ is the Shahshahani mutation/selection potential under the house of cards condition for mutations (24)

$$
V(\mathbf{x})=\log \mathcal{W}(\mathbf{x}), \text { where } \mathcal{W}(\mathbf{x})=e^{-|\boldsymbol{\mu}|} \prod_{k=1}^{K} x_{k}^{\mu_{k}}\left(\mathbf{x}^{\prime} W \mathbf{x}\right)^{(1-|\boldsymbol{\mu}|) / 2},
$$

then, with

$$
\begin{gathered}
Z_{N}(2 N|\boldsymbol{\mu}|)=\int_{\mathcal{S}_{K}} d \mathbf{x} \prod_{k=1}^{K} x_{k}^{2 N \mu_{k}-1}\left(\mathbf{x}^{\prime} W \mathbf{x}\right)^{N(1-|\boldsymbol{\mu}|)}<\infty \\
p_{N}(\mathbf{x})=\frac{1}{Z_{N}(2 N|\boldsymbol{\mu}|)} \prod_{k=1}^{K} x_{k}^{2 N \mu_{k}-1}\left(\mathbf{x}^{\prime} W \mathbf{x}\right)^{N(1-|\boldsymbol{\mu}|)} .
\end{gathered}
$$

If $|\boldsymbol{\mu}|=1$, we recognize the skew Dirichlet distribution $D_{K}$ on the simplex $\mathcal{S}_{K}$, with parameters $2 N \mu_{k}, k=1, \ldots, K$ :

$$
p_{N}(\mathbf{x})=\frac{1}{Z_{N}^{D}(2 N \boldsymbol{\mu})} \prod_{k=1}^{K} x_{k}^{2 N \mu_{k}-1}, \mathbf{x} \in \mathcal{S}_{K},
$$

\footnotetext{
${ }^{4}$ In the sequel, we consider a mean fitness $\mathbf{x}^{\prime} W \mathbf{x}$ but a frequency-dependent mean fitness $\mathbf{x}^{\prime} W(\mathbf{x}) \mathbf{x}$ would do as well provided $\mathbf{x}^{\prime} W(\mathbf{x}) \mathbf{x}$ is bounded as $\mathbf{x} \in S_{K}$.
} 
with normalizing partition function $Z_{N}^{D}(2 N \boldsymbol{\mu})=\prod_{k=1}^{K} \Gamma\left(2 N \mu_{k}\right) / \Gamma(2 N|\boldsymbol{\mu}|)$. We shall call $D_{K}(2 N \boldsymbol{\mu})$ this distribution so that here $|\boldsymbol{\mu}|=1 \Rightarrow \mathbf{X}_{N} \sim D_{K}(2 N \boldsymbol{\mu})$. Except in the homogeneous case when all $\mu_{k}=\mu$ for each $k=1, \ldots, K$, Dirichlet distributed random variables with distribution (30) on the simplex are not exchangeable and $p_{N}(\mathbf{x})$ is not invariant under a permutation of its coordinates, [23]. The same holds a fortiori for (29) when $|\boldsymbol{\mu}| \neq 1$.

With $V$ as in (28), the deterministic dynamics corresponding to this particular potential is thus $\Delta \mathbf{x}=\varepsilon G(\mathbf{x}) \nabla V(\mathbf{x})=: \varepsilon \mathbf{f}(\mathbf{x})$, as a version of (23) when the time elapsed between two consecutive generations no longer is 1 but $\varepsilon$.

5.1.5. Weak mutation probabilities. If $2 N \mu_{k}=\theta_{k}$ (small mutation probabilities, with all $\theta_{k}>0$ now defining mutation rates), with $\boldsymbol{\theta}^{\prime}:=\left(\theta_{1}, \ldots, \theta_{K}\right)$ and $|\boldsymbol{\theta}|:=\sum_{k=1}^{K} \theta_{k}$

$$
\mathbf{X}_{N} \sim p_{N}(\mathbf{x})=\frac{1}{Z_{N}(\boldsymbol{\theta})} \prod_{k=1}^{K} x_{k}^{\theta_{k}-1}\left(\mathbf{x}^{\prime} W \mathbf{x}\right)^{N-|\boldsymbol{\theta}| / 2}
$$

where

$$
Z_{N}(\boldsymbol{\theta})=\int_{\mathcal{S}_{K}} d \mathbf{x} \prod_{k=1}^{K} x_{k}^{\theta_{k}-1}\left(\mathbf{x}^{\prime} W \mathbf{x}\right)^{N-|\boldsymbol{\theta}| / 2}<\infty .
$$

In the asymmetric mutation case when $\boldsymbol{\theta}^{\prime} \neq \theta \mathbf{1}^{\prime}$ for some common $\theta>0$, the probability distribution of $\mathbf{X}_{N}$ on the simplex is not exchangeable either. For similar shapes of the equilibrium distribution in this weak mutation setup, see [37], [24, 25] and 38.

With $\mathcal{Z}_{N}(\boldsymbol{\theta}):=Z_{N}(\boldsymbol{\theta}) / Z^{D}(\boldsymbol{\theta}), Z^{D}(\boldsymbol{\theta}):=\prod_{k=1}^{K} \Gamma\left(\theta_{k}\right) / \Gamma(|\boldsymbol{\theta}|)$, (31) is also

$$
p_{N}(\mathbf{x})=\frac{1}{\mathcal{Z}_{N}(\boldsymbol{\theta}) Z^{D}(\boldsymbol{\theta})} \prod_{k=1}^{K} x_{k}^{\theta_{k}-1}\left(\mathbf{x}^{\prime} W \mathbf{x}\right)^{N-|\boldsymbol{\theta}| / 2}
$$

where

$$
\mathcal{Z}_{N}(\boldsymbol{\theta})=\frac{1}{Z^{D}(\boldsymbol{\theta})} \int_{\mathcal{S}_{K}} \prod_{k=1}^{K} x_{k}^{\theta_{k}-1}\left(\mathbf{x}^{\prime} W \mathbf{x}\right)^{N-|\boldsymbol{\theta}| / 2}=\mathbf{E}\left(\mathbf{S}^{\prime} W \mathbf{S}\right)^{N-|\boldsymbol{\theta}| / 2} .
$$

and $\mathbf{S} \sim D_{K}(\boldsymbol{\theta})$ is Dirichlet distributed.

5.1.6. Weak mutation and weak selection probabilities. If in addition $W=$ $J-\frac{1}{N} \bar{W}$ for some new symmetric fitness differential matrix $\bar{W} \geq \mathbf{0}$ (involving an order $N^{-1}$ correction to the neutral model $J$ of selection), then $\mathbf{x}^{\prime} W \mathbf{x}=1-$ $N^{-1} \mathbf{x}^{\prime} \bar{W} \mathbf{x}$ and

$$
\left(\mathbf{x}^{\prime} W \mathbf{x}\right)^{N-|\boldsymbol{\theta}| / 2} \sim e^{-\mathbf{x}^{\prime} \bar{W} \mathbf{x}} \text { for large } N
$$

so that

$$
p(\mathbf{x})=\frac{1}{Z(\boldsymbol{\theta})} \prod_{k=1}^{K} x_{k}^{\theta_{k}-1} e^{-\mathbf{x}^{\prime} \bar{W} \mathbf{x}},
$$

for some normalizing constant

$$
Z(\boldsymbol{\theta})=\int_{\mathcal{S}_{K}} d \mathbf{x} \prod_{k=1}^{K} x_{k}^{\theta_{k}-1} e^{-\mathbf{x}^{\prime} \bar{W} \mathbf{x}}
$$


With $Z^{D}(\boldsymbol{\theta}):=\prod_{k=1}^{K} \Gamma\left(\theta_{k}\right) / \Gamma(|\boldsymbol{\theta}|)=B_{k}(\boldsymbol{\theta})$ (the multidimensional beta function) and $\mathcal{Z}(\boldsymbol{\theta}):=Z(\boldsymbol{\theta}) / Z^{D}(\boldsymbol{\theta})$, we have

$$
p(\mathbf{x})=\frac{1}{\mathcal{Z}(\boldsymbol{\theta}) Z^{D}(\boldsymbol{\theta})} \prod_{k=1}^{K} x_{k}^{\theta_{k}-1} e^{-\mathbf{x}^{\prime} \bar{W} \mathbf{x}},
$$

where

$$
\mathcal{Z}(\boldsymbol{\theta})=\frac{1}{Z^{D}(\boldsymbol{\theta})} \int_{\mathcal{S}_{K}} \prod_{k=1}^{K} x_{k}^{\theta_{k}-1} e^{-\mathbf{x}^{\prime} \bar{W} \mathbf{x}}=\mathbf{E}\left(e^{-\mathbf{S}^{\prime} \bar{W} \mathbf{S}}\right)
$$

with $\mathbf{S} \sim D_{K}(\boldsymbol{\theta})$. The latter $p(\mathbf{x})$ is the unique invariant probability density of the diffusion process on the simplex, [32, 7]

$$
d \mathbf{x}_{t}=\mathbf{f}\left(\mathbf{x}_{t}\right) d t+G^{1 / 2}\left(\mathbf{x}_{t}\right) d \mathbf{w}_{t},
$$

where the drift $\mathbf{f}(\mathbf{x})$ is

$$
\begin{aligned}
& \mathbf{f}(\mathbf{x})=G(\mathbf{x}) \nabla \bar{V}(\mathbf{x}) \text { with } \\
& \bar{V}(\mathbf{x})=\log \overline{\mathcal{W}}(\mathbf{x}), \text { and } \overline{\mathcal{W}}(\mathbf{x})=\left(\prod_{k=1}^{K} x_{k}^{\theta_{k}}\right) e^{-\mathbf{x}^{\prime} \bar{W} \mathbf{x}} .
\end{aligned}
$$

Namely,

$$
\begin{aligned}
& p(\mathbf{x})=\frac{1}{Z(\boldsymbol{\theta})} \operatorname{det}\left(G^{-1}\right) \exp \int^{\mathbf{x}}\left(G^{-1} \mathbf{f}\right)(\mathbf{y}) \cdot d \mathbf{y} \\
& =\frac{1}{Z(\boldsymbol{\theta})}\left(\prod_{k=1}^{K} x_{k}^{-1}\right) \overline{\mathcal{W}}(\mathbf{x})=\frac{1}{Z(\boldsymbol{\theta})} \prod_{k=1}^{K} x_{k}^{\theta_{k}-1} e^{-\mathbf{x}^{\prime} \bar{W} \mathbf{x}} .
\end{aligned}
$$

A unit time $t$ in the latter diffusion process represents $N$ generations of the discretetime model. See [37], 25], [12] and [1].

\subsection{Computing the partition functions $Z_{N}(\boldsymbol{\theta})$ and $Z(\boldsymbol{\theta})$.}

\subsubsection{A constructive formula for computing with Dirichlet $(\theta)$ distribu-} tion. The following 'Gamma-calculus' result will be useful (see [18]):

Theorem 1. Consider an asymmetric Dirichlet distributed random variable on the $(K-1)$-simplex, viz: $\mathbf{S} \stackrel{d}{\sim} D_{K}(\boldsymbol{\theta})$ and let $\mathbf{S}(t)=t \mathbf{S}(1)=t \mathbf{S}=\left(t S_{1}, \ldots, t S_{K}\right)$, $t>0$, with $\sum_{k=1}^{K} S_{k}(t)=t$.

(i) Let $f$ be any Borel-measurable function for which

$$
\int_{0}^{\infty} \mathbf{E}(|f(\mathbf{S}(t))|) t^{|\boldsymbol{\theta}|-1} e^{-p t} d t<\infty, p>0 .
$$

Then, with $\mathbf{T}(p):=\left(T_{k}(p) ; k=1, \ldots, K\right), K$ independent random variables defined by $T_{k}(p)=\frac{1}{p} T_{k}, p>0, k=1, \ldots, K$ where $T_{k}(1):=T_{k} \stackrel{d}{\sim} \operatorname{gamma}\left(\theta_{k}\right)$, we have

$$
\int_{0}^{\infty} \mathbf{E}(f(\mathbf{S}(t))) t^{|\boldsymbol{\theta}|-1} e^{-p t} d t=\frac{\Gamma(|\boldsymbol{\theta}|)}{p^{|\boldsymbol{\theta}|}} \mathbf{E}(f(\mathbf{T}(p))) .
$$

(ii) If $f$ is homogeneous of degree $d$, i.e. if $f(t \mathbf{s})=t^{d} f(\mathbf{s}), t>0, \mathbf{s}:=\left(s_{1}, \ldots, s_{K}\right) \in$ $\mathbf{R}^{n}$, and if $\mathbf{E}(|f(\mathbf{S})|)<\infty$ then, with $\mathbf{T}^{\prime}:=\left(T_{1}, \ldots, T_{K}\right)$,

$$
\mathbf{E}(f(\mathbf{S}))=\frac{\Gamma(|\boldsymbol{\theta}|)}{\Gamma(|\boldsymbol{\theta}|+d)} \mathbf{E}(f(\mathbf{T})) .
$$


This shows that computing the expected value of some functional with respect to the asymmetric Dirichlet distribution on the simplex can be achieved while averaging over an identically distributed gamma distributed sample; a much simpler task.

\subsubsection{Estimating $Z_{N}(\theta)$ in (32).}

Theorem 2. When $N \gg|\boldsymbol{\theta}| / 2$,

$$
Z_{N}(\boldsymbol{\theta}) \sim \frac{N !}{\Gamma(2 N)} \sum_{|[\mathbf{n}]|=N} \prod_{k, l=1}^{K} \frac{W_{k, l}^{n_{k, l}}}{n_{k, l} !} \prod_{k=1}^{K} \Gamma\left(\theta_{k}+\alpha_{k}([\mathbf{n}])\right),
$$

where the summation runs over all integer ordered partitions $[\mathbf{n}]:=n_{k, l}$ of $N$ as $|[\mathbf{n}]|:=\sum_{k, l=1}^{K} n_{k, l}=N$ and $\alpha_{k}([\mathbf{n}])=\sum_{l=1}^{K}\left(n_{k, l}+n_{l, k}\right)$.

Proof: Consider the statement (ii) of Theorem 1 with $f(\mathbf{S})=\left(\mathbf{S}^{\prime} \bar{W} \mathbf{S}\right)^{N-|\boldsymbol{\theta}| / 2}$. This function $f$ is homogeneous of degree $d=2 N-|\boldsymbol{\theta}|$. Thus

$$
\mathcal{Z}_{N}(\boldsymbol{\theta})=\mathbf{E}\left(\mathbf{S}^{\prime} W \mathbf{S}\right)^{N-|\boldsymbol{\theta}| / 2}=\frac{\Gamma(|\boldsymbol{\theta}|)}{\Gamma(2 N)} \mathbf{E}\left(\mathbf{T}^{\prime} W \mathbf{T}\right)^{N-|\boldsymbol{\theta}| / 2}
$$

where the random vector $\mathbf{T}$ has independent components with $T_{k} \stackrel{d}{\sim} \operatorname{gamma}\left(\theta_{k}\right)$. Thus, with $\mathbf{t}^{\prime}=\left(t_{1}, \ldots, t_{K}\right)$

$$
\begin{aligned}
& \mathcal{Z}_{N}(\boldsymbol{\theta})=\frac{\Gamma(|\boldsymbol{\theta}|)}{\prod_{k=1}^{K} \Gamma\left(\theta_{k}\right)} \frac{1}{\Gamma(2 N)} \int_{\mathbf{R}_{+}^{K}}\left(\mathbf{t}^{\prime} W \mathbf{t}\right)^{N-|\boldsymbol{\theta}| / 2} \prod_{k=1}^{K} t_{k}^{\theta_{k}-1} e^{-t_{k}} d t_{k} \text { or } \\
& Z_{N}(\boldsymbol{\theta})=\frac{1}{\Gamma(2 N)} \int_{\mathbf{R}_{+}^{K}}\left(\mathbf{t}^{\prime} W \mathbf{t}\right)^{N-|\boldsymbol{\theta}| / 2} \prod_{k=1}^{K} t_{k}^{\theta_{k}-1} e^{-t_{k}} d t_{k} .
\end{aligned}
$$

When $N$ is large enough, $\mathcal{Z}_{N}(\boldsymbol{\theta})$ is close to $\frac{\Gamma(|\boldsymbol{\theta}|)}{\Gamma(2 N)} \mathbf{E}\left(\mathbf{T}^{\prime} W \mathbf{T}\right)^{N}$, with

$$
\mathbf{E}\left(\mathbf{T}^{\prime} W \mathbf{T}\right)^{N}=N ! \sum_{|[\mathbf{n}]|=N} \prod_{k, l=1}^{K} \frac{W_{k, l}^{n_{k} l}}{n_{k, l} !} \prod_{k=1}^{K} \mathbf{E}\left(T_{k}^{\alpha_{k}([\mathbf{n}])}\right) .
$$

In the latter expression, the summation runs over all square $K \times K$ arrays $[\mathbf{n}]_{k: l}:=n_{k, l}$ whose integral non-negative entries sum to $N$ (the set $\{[\mathbf{n}]:|[\mathbf{n}]|=N\}$ ) 5 , and $\alpha_{k}([\mathbf{n}]):=\sum_{l=1}^{K}\left(n_{k, l}+n_{l, k}\right)$, integers. Recalling the moment structure of gamma random variables $(\mathrm{rvs}), \mathbf{E}\left(T_{k}^{\alpha_{k}([\mathbf{n}])}\right)=\frac{\Gamma\left(\theta_{k}+\alpha_{k}([\mathbf{n}])\right)}{\Gamma\left(\theta_{k}\right)}$, we thus get

$$
\begin{aligned}
& \mathcal{Z}_{N}(\boldsymbol{\theta}) \sim \frac{\Gamma(|\boldsymbol{\theta}|)}{\Gamma(2 N)} N ! \sum_{|[\mathbf{n}]|=N} \prod_{k, l=1}^{K} \frac{W_{k, l}^{n_{k} l}}{n_{k, l} !} \prod_{k=1}^{K} \frac{\Gamma\left(\theta_{k}+\alpha_{k}([\mathbf{n}])\right)}{\Gamma\left(\theta_{k}\right)} \\
& Z_{N}(\boldsymbol{\theta}) \sim \frac{N !}{\Gamma(2 N)} \sum_{|[\mathbf{n}]|=N} \prod_{k, l=1}^{K} \frac{W_{k, l}^{n_{k, l}}}{n_{k, l} !} \prod_{k=1}^{K} \Gamma\left(\theta_{k}+\alpha_{k}([\mathbf{n}])\right) .
\end{aligned}
$$

\footnotetext{
${ }^{5}$ The number of ordered partitions of the integer $N$ into $k$ non-negative integral summands, $k=$ $1, \ldots, K^{2}$, is $\left(\begin{array}{c}N+k-1 \\ k-1\end{array}\right)$. There are thus $\sum_{k=1}^{K^{2}}\left(\begin{array}{c}N+k-1 \\ k-1\end{array}\right)=\left(\begin{array}{c}N+K^{2}-1 \\ K^{2}-1\end{array}\right) \underset{N \text { large }}{\sim} N^{K^{2}-1} /\left(K^{2}-1\right)$ ! ways to realize $|[\mathbf{n}]|:=\sum_{k, l=1}^{K} n_{k, l}=N$, with $n_{k, l}$ non-negative integers.
} 


\subsubsection{Computing $Z(\boldsymbol{\theta})$ in (34). We now show that}

\section{Theorem 3.}

$$
\begin{aligned}
& Z(\boldsymbol{\theta})=\frac{\prod_{k=1}^{K} \Gamma\left(\theta_{k}\right)}{\Gamma(|\boldsymbol{\theta}|)} \times \\
& \left(1+\frac{\Gamma(|\boldsymbol{\theta}|)}{\prod_{k=1}^{K} \Gamma\left(\theta_{k}\right)} \sum_{N \geq 1}(-1)^{N} \sum_{|[\mathbf{n}]|=N} \frac{\prod_{k=1}^{K} \Gamma\left(\theta_{k}+\alpha_{k}([\mathbf{n}])\right)}{\Gamma(|\boldsymbol{\theta}|+2 N)} \prod_{k, l=1}^{K} \frac{\bar{W}_{k, l}^{n_{k, l}}}{n_{k, l} !}\right) .
\end{aligned}
$$

Proof: Consider the statement (i) of Theorem 1. From (38), the right hand-side quantity, namely

$$
\Gamma(|\boldsymbol{\theta}|) p^{-|\boldsymbol{\theta}|} \mathbf{E}(f(\mathbf{T}(p)))
$$

may be interpreted as the Laplace transform in the variable $p$ of $\mathbf{E}(f(\mathbf{S}(t))) t^{|\boldsymbol{\theta}|-1}$ appearing in the left-hand side. Inverting this Laplace transform and putting $t=1$ yields $\mathbf{E}(f(\mathbf{S}))$. This can be used to compute $\mathcal{Z}(\boldsymbol{\theta})=\mathbf{E}\left(e^{-\mathbf{S}^{\prime} \bar{W} \mathbf{S}}\right)$ with $f(\mathbf{S})=$ $e^{-\mathbf{S}^{\prime} \bar{W} \mathbf{S}}$. We have

$$
\mathbf{E}\left(e^{-\mathbf{T}^{\prime}(p) \bar{W} \mathbf{T}(p)}\right)=1+\sum_{N \geq 1} \frac{(-1)^{N}}{N !} \mathbf{E}\left(\mathbf{T}^{\prime}(p) \bar{W} \mathbf{T}(p)^{N}\right),
$$

where,

$$
\mathbf{E}\left(\mathbf{T}^{\prime}(p) \bar{W} \mathbf{T}(p)^{N}\right)=N ! \sum_{|[\mathbf{n}]|=N} \prod_{k, l=1}^{K} \frac{\bar{W}_{k, l}^{n_{k, l}}}{n_{k, l} !} \prod_{k=1}^{K} \mathbf{E}\left(T_{k}(p)^{\alpha_{k}([\mathbf{n}])}\right) .
$$

Recalling $\mathbf{E}\left(T_{k}(p)^{\alpha_{k}([\mathbf{n}])}\right)=\frac{\Gamma\left(\theta_{k}+\alpha_{k}([\mathbf{n}])\right)}{\Gamma\left(\theta_{k}\right)} p^{-\alpha_{k}([\mathbf{n}])}$, we get

$$
\mathbf{E}\left(e^{-\mathbf{T}^{\prime}(p) \bar{W} \mathbf{T}(p)}\right)=1+\sum_{N \geq 1}(-1)^{N} \sum_{|[\mathbf{n}]|=N} \prod_{k, l=1}^{K} \frac{\bar{W}_{k, l}^{n_{k, l}}}{n_{k, l} !} \prod_{k=1}^{K} \frac{\Gamma\left(\theta_{k}+\alpha_{k}([\mathbf{n}])\right)}{\Gamma\left(\theta_{k}\right)} p^{-\alpha_{k}([\mathbf{n}])} .
$$

Owing to $\sum_{k=1}^{K} \alpha_{k}([\mathbf{n}])=2 N$, this is also

$$
\mathbf{E}\left(e^{-\mathbf{T}^{\prime}(p) \bar{W} \mathbf{T}(p)}\right)=1+\sum_{N \geq 1} p^{-2 N} \sum_{|[\mathbf{n}]|=N}(-1)^{N} \prod_{k, l=1}^{K} \frac{\bar{W}_{k, l}^{n_{k}, l}}{n_{k, l} !} \prod_{k=1}^{K} \frac{\Gamma\left(\theta_{k}+\alpha_{k}([\mathbf{n}])\right)}{\Gamma\left(\theta_{k}\right)} .
$$

The right hand-side quantity of statement (38) is thus

$$
\begin{gathered}
\Gamma(|\boldsymbol{\theta}|) p^{-|\boldsymbol{\theta}|} \mathbf{E}(f(\mathbf{T}(p)))= \\
\Gamma(|\boldsymbol{\theta}|) p^{-|\boldsymbol{\theta}|}+\Gamma(|\boldsymbol{\theta}|) \sum_{N \geq 1} p^{-(2 N+|\boldsymbol{\theta}|)} \sum_{|[\mathbf{n}]|=N}(-1)^{N} \prod_{k, l=1}^{K} \frac{\bar{W}_{k, l}^{n_{k, l}}}{n_{k, l} !} \prod_{k=1}^{K} \frac{\Gamma\left(\theta_{k}+\alpha_{k}([\mathbf{n}])\right)}{\Gamma\left(\theta_{k}\right)} .
\end{gathered}
$$

It is the Laplace-Stieltjes transform of $\mathbf{E}\left(e^{-\mathbf{S}^{\prime}(t) \bar{W} \mathbf{S}(t)}\right) t^{|\boldsymbol{\theta}|-1}$.

But $p^{-(2 N+|\boldsymbol{\theta}|)}$ is the Laplace-Stieltjes transform of $t^{2 N+|\boldsymbol{\theta}|-1} / \Gamma(2 N+|\boldsymbol{\theta}|)$ and $p^{-|\boldsymbol{\theta}|}$ the one of $t^{|\boldsymbol{\theta}|-1} / \Gamma(|\boldsymbol{\theta}|)$. Inverting the Laplace transform and evaluating the result at $t=1$ yields

$$
\begin{aligned}
& \mathcal{Z}(\boldsymbol{\theta}):=\mathbf{E}\left(e^{-\mathbf{S}^{\prime} \bar{W} \mathbf{S}}\right) \\
& =1+\sum_{N \geq 1}(-1)^{N} \frac{\Gamma(|\boldsymbol{\theta}|)}{\Gamma(2 N+|\boldsymbol{\theta}|)} \sum_{|[\mathbf{n}]|=N} \prod_{k, l=1}^{K} \frac{\bar{W}_{k, l}^{n_{k}, l}}{n_{k, l} !} \prod_{k=1}^{K} \frac{\Gamma\left(\theta_{k}+\alpha_{k}([\mathbf{n}])\right)}{\Gamma\left(\theta_{k}\right)} \\
& =1+\frac{1}{Z^{D}(\boldsymbol{\theta})} \sum_{N \geq 1}(-1)^{N} \sum_{|[\mathbf{n}]|=N} \frac{\prod_{k=1}^{K} \Gamma\left(\theta_{k}+\alpha_{k}([\mathbf{n}])\right)}{\Gamma(|\boldsymbol{\theta}|+2 N)} \prod_{k, l=1}^{K} \frac{\bar{W}_{k, l}^{n}, n_{k, l}}{n_{k, l} !} .
\end{aligned}
$$


Recalling $Z(\boldsymbol{\theta})=Z^{D}(\boldsymbol{\theta}) \mathcal{Z}(\boldsymbol{\theta})$, we thus obtain

$$
\begin{aligned}
& Z(\boldsymbol{\theta})=Z^{D}(\boldsymbol{\theta}) \mathbf{E}\left(e^{-\mathbf{S}^{\prime} \bar{W} \mathbf{S}}\right) \\
& =Z^{D}(\boldsymbol{\theta})\left(1+\frac{1}{Z^{D}(\boldsymbol{\theta})} \sum_{N \geq 1}(-1)^{N} \sum_{|[\mathbf{n}]|=N} \frac{\prod_{k=1}^{K} \Gamma\left(\theta_{k}+\alpha_{k}([\mathbf{n}])\right)}{\Gamma(|\boldsymbol{\theta}|+2 N)} \prod_{k, l=1}^{K} \frac{\bar{W}_{k, l}^{n_{k, l}}}{n_{k, l} !}\right) .
\end{aligned}
$$

Corollary 4. The alternating series expansion of $\mathcal{Z}(\boldsymbol{\theta})$ in $\sqrt{42)}$ is convergent.

Proof: Let

$$
u_{N}(\boldsymbol{\theta}):=\frac{1}{Z^{D}(\boldsymbol{\theta})} \sum_{|[\mathbf{n}]|=N} \frac{\prod_{k=1}^{K} \Gamma\left(\theta_{k}+\alpha_{k}([\mathbf{n}])\right)}{\Gamma(|\boldsymbol{\theta}|+2 N)} \prod_{k, l=1}^{K} \frac{\bar{W}_{k, l}^{n_{k, l}}}{n_{k, l} !}
$$

so that $\mathcal{Z}(\boldsymbol{\theta})=1+\sum_{N \geq 1}(-1)^{N} u_{N}(\boldsymbol{\theta})$. Let $\bar{w}>0$ be the largest of the $\bar{W}_{k, l}$ s. Then $\prod_{k, l=1}^{K} \bar{W}_{k, l}^{n_{k, l}}<\bar{w}^{N}$ and

$$
u_{N}(\boldsymbol{\theta})<\bar{w}^{N} \frac{1}{Z^{D}(\boldsymbol{\theta})} \sum_{|\mathbf{n}|=N} \frac{\prod_{k=1}^{K} \Gamma\left(\theta_{k}+2 n_{k}\right)}{\Gamma(|\boldsymbol{\theta}|+2 N)} \prod_{k=1}^{K} \frac{1}{n_{k} !},
$$

where $\mathbf{n}=\left(n_{1}, \ldots, n_{K}\right)^{\prime}$ is now a vector with nonnegative integral entries summing to $N$. With $\mathbf{S} \sim D_{K}(\boldsymbol{\theta})$, we now have

$$
\frac{1}{Z^{D}(\boldsymbol{\theta})} \frac{\prod_{k=1}^{K} \Gamma\left(\theta_{k}+2 n_{k}\right)}{\Gamma(|\boldsymbol{\theta}|+2 N)}=\mathbf{E}\left(\prod_{k=1}^{K} S_{k}^{2 n_{k}}\right)<1,
$$

which are the integral moments of order $2 \mathbf{n}$ of a $\operatorname{Dirichlet}(\boldsymbol{\theta})$ random vector (see (58) below). Thus, $u_{N}(\boldsymbol{\theta})<\frac{(K \bar{w})^{N}}{N !}$ and $\mathcal{Z}(\boldsymbol{\theta})$ is absolutely convergent hence convergent.

Remarks: As $N$ gets large, the contribution of $u_{N}(\boldsymbol{\theta})$ to $\mathcal{Z}(\boldsymbol{\theta})$ becomes rapidly smaller and smaller suggesting that only a few first terms of the series-expansion of $\mathcal{Z}(\boldsymbol{\theta})$ should lead to a satisfactory approximation. When $N=1$, the first-order term $-u_{1}(\boldsymbol{\theta})$ is

$$
-u_{1}(\boldsymbol{\theta})=\frac{-1}{Z^{D}(\boldsymbol{\theta})} \frac{\prod_{k=1}^{K} \Gamma\left(\theta_{k}\right)}{\Gamma(|\boldsymbol{\theta}|+2)} \sum_{k, l=1}^{K} \theta_{k} \bar{W}_{k, l} \theta_{l}=\frac{-1}{|\boldsymbol{\theta}|(|\boldsymbol{\theta}|+1)} \boldsymbol{\theta}^{\prime} \bar{W} \boldsymbol{\theta} .
$$

Note finally that, with $\boldsymbol{\alpha}([\mathbf{n}]):=\left(\alpha_{1}([\mathbf{n}]), \ldots, \alpha_{K}([\mathbf{n}])\right)^{\prime}$ and $\boldsymbol{\theta}+\boldsymbol{\alpha}([\mathbf{n}])=\theta_{k}+$ $\alpha_{k}([\mathbf{n}]) ; k=1, \ldots, K$, the factors

$$
Z^{D}(\boldsymbol{\theta}+\boldsymbol{\alpha}([\mathbf{n}]))=\frac{\prod_{k=1}^{K} \Gamma\left(\theta_{k}+\alpha_{k}([\mathbf{n}])\right)}{\Gamma(|\boldsymbol{\theta}|+2 N)},
$$

appearing in the series expansion of $Z(\boldsymbol{\theta})$ are the normalizing constants of a $D_{K}(\boldsymbol{\theta}+\boldsymbol{\alpha}([\mathbf{n}]))$ Dirichlet distribution $\diamond$.

\section{Generalized Ewens sampling formulae}

6.1. The weak mutation/selection potential case. Let

$$
\mathbf{X} \sim p(\mathbf{x})=\frac{1}{Z(\boldsymbol{\theta})} \prod_{k=1}^{K} x_{k}^{\theta_{k}-1} e^{-\mathbf{x}^{\prime} \bar{W} \mathbf{x}}
$$


whose support is the simplex $\mathcal{S}_{K}$. We wish to consider sampling problems within $\mathbf{X}$ (as a random partition of the unit interval $[0,1]$ ) describing the equilibrium distribution of the allelic frequencies, 29. Such generalized Ewens sampling formulae (ESF) were also considered in [34]-[36], in this context.

6.1.1. Moments. We first consider the simpler problem of computing the moments of $\mathbf{X}$. With $\mathbf{m}^{\prime}:=\left(m_{1}, \ldots, m_{K}\right)$ non-negative integers, the $\mathbf{m}$-moments of this stationary distribution are:

$$
\mathbf{E}\left(\prod_{k=1}^{K} X_{k}^{m_{k}}\right)=\frac{\int_{\mathcal{S}_{K}} d \mathbf{x} \prod_{k=1}^{K} x_{k}^{\theta_{k}+m_{k}-1} e^{-\mathbf{x}^{\prime} \bar{W} \mathbf{x}}}{\int_{\mathcal{S}_{K}} d \mathbf{x} \prod_{k=1}^{K} x_{k}^{\theta_{k}-1} e^{-\mathbf{x}^{\prime} \bar{W} \mathbf{x}}}=\frac{\mathcal{Z}(\boldsymbol{\theta}+\mathbf{m})}{\mathcal{Z}(\boldsymbol{\theta})},
$$

requiring the previous computation of $\mathcal{Z}(\boldsymbol{\theta})$. With $m=\sum_{k=1}^{K} m_{k}$, we find from Equation (42)

$$
\begin{aligned}
& \mathbf{E}\left(\prod_{k=1}^{K} X_{k}^{m_{k}}\right)= \\
& \frac{1+\frac{\Gamma(|\boldsymbol{\theta}|+m)}{\prod_{k=1}^{K} \Gamma\left(\theta_{k}+m_{k}\right)} \sum_{N \geq 1}(-1)^{N} \sum_{|[\mathbf{n}]|=N} \frac{\prod_{k=1}^{K} \Gamma\left(\theta_{k}+m_{k}+\alpha_{k}([\mathbf{n}])\right)}{\Gamma(|\boldsymbol{\theta}|+m+2 N)} \prod_{k, l=1}^{K} \frac{\bar{W}_{k, l}^{n_{k}, l}}{n_{k, l} !}}{1+\frac{\Gamma(|\boldsymbol{\theta}|)}{\prod_{k=1}^{K} \Gamma\left(\theta_{k}\right)} \sum_{N \geq 1}(-1)^{N} \sum_{|[\mathbf{n}]|=N} \frac{\prod_{k=1}^{K} \Gamma\left(\theta_{k}+\alpha_{k}([\mathbf{n}])\right)}{\Gamma(\boldsymbol{\theta} \mid+2 N)} \prod_{k, l=1}^{K} \frac{\bar{W}_{k, l}^{n_{k}, l}}{n_{k, l} l^{!}}} .
\end{aligned}
$$

Note that, from the identity

$$
\begin{gathered}
\mathbf{E}\left[\left(\sum_{k=1}^{K} u_{k} X_{k}\right)^{m}\right]=\sum_{|\mathbf{m}|=m}\left(\begin{array}{c}
m \\
m_{1} \ldots m_{K}
\end{array}\right) \mathbf{E}\left(\prod_{k=1}^{K} X_{k}^{m_{k}}\right) \prod_{k=1}^{K} u_{k}^{m_{k}}, \\
\left(\begin{array}{c}
m \\
m_{1} \ldots m_{K}
\end{array}\right) \mathbf{E}\left(\prod_{k=1}^{K} X_{k}^{m_{k}}\right)=\left[\prod_{k=1}^{K} u_{k}^{m_{k}}\right] \mathbf{E}\left[\left(\sum_{k=1}^{K} u_{k} X_{k}\right)^{m}\right],
\end{gathered}
$$

where $\left(\sum_{k=1}^{K} u_{k} X_{k}\right)^{m}$ is homogeneous of degree $m$. In particular

$$
1=\mathbf{E}\left[\left(X_{1}+\ldots+X_{K}\right)^{m}\right]=\sum_{|\mathbf{m}|=m}\left(\begin{array}{c}
m \\
m_{1} \ldots m_{K}
\end{array}\right) \mathbf{E}\left(\prod_{k=1}^{K} X_{k}^{m_{k}}\right) .
$$

The marginal moments $\mathbf{E}\left(X_{l}^{m_{l}}\right)$ are obtained from $\mathbf{E}\left(\prod_{k=1}^{K} X_{k}^{m_{k}}\right)$ in (45), while considering $m_{k}=0$ except for $k=l$.

6.1.2. Generalized Ewens Sampling Formula. Take a random (uniform) sequential $m$-sample without replacement from $\mathbf{X} \sim p(\mathbf{x})$, describing the random equilibrium distribution of the allelic frequencies. Suppose there are $P_{K, m}=p$ distinct visited types of alleles in the process; suppose also that $K_{1}=k_{1}, \ldots, K_{p}=k_{p} \in$ $\{1, \ldots, K\}^{p}$ are the types of the visited alleles and that $B_{K, m}\left(k_{1}\right)=m_{1}, . ., B_{K, m}\left(k_{p}\right)=$ $m_{p}$ are the number of visits to (or hits of) alleles number $k_{1}, \ldots, k_{p}$, entailing $m_{1}, . ., m_{p} \geq 1$ and $m=m_{1}+\ldots+m_{p}$. We let $\boldsymbol{\theta}+\mathbf{m}_{p}:=\theta_{k_{q}}+m_{q} ; q=1, \ldots, p$ shifting only the $\theta_{k_{q}}$-entries of $\boldsymbol{\theta}$ by $m_{q}$ and leaving the other ones unchanged. From (44) and (42), we obtain:

Theorem 5. With $m_{1}, . ., m_{p} \geq 1$ summing to $m$ and $p \leq m \wedge K$, the probability of such an occupancy event is

$$
\mathbf{P}\left(K_{1}=k_{1}, \ldots, K_{p}=k_{p} ; B_{K, m}\left(k_{1}\right)=m_{1}, . ., B_{K, m}\left(k_{p}\right)=m_{p} ; P_{K, m}=p\right)
$$




$$
\begin{aligned}
& =\mathbf{E}\left(\prod_{q=1}^{p} X_{k_{q}}^{m_{q}}\right)=\frac{\mathcal{Z}\left(\boldsymbol{\theta}+\mathbf{m}_{p}\right)}{\mathcal{Z}(\boldsymbol{\theta})}:=\frac{Z^{D}(\boldsymbol{\theta})^{-1} \int_{\mathcal{S}_{K}} d \mathbf{x} \prod_{q=1}^{p} x_{k_{q}}^{\theta_{k_{q}}+m_{q}-1} \prod_{k \neq\left\{k_{1}, \ldots, k_{p}\right\}} x_{k}^{\theta_{k}-1} e^{-\mathbf{x}^{\prime} \bar{W} \mathbf{x}}}{Z^{D}(\boldsymbol{\theta})^{-1} \int_{\mathcal{S}_{K}} d \mathbf{x} \prod_{k=1}^{K} x_{k}^{\theta_{k}-1} e^{-\mathbf{x}^{\prime} \bar{W} \mathbf{x}}} \\
& =\frac{1}{\mathcal{Z}(\boldsymbol{\theta})}\left(1+\frac{\Gamma(|\boldsymbol{\theta}|+m)}{\prod_{q=1}^{p} \Gamma\left(\theta_{k_{q}}+m_{q}\right) \prod_{k \neq\left\{k_{1}, \ldots, k_{p}\right\}} \Gamma\left(\theta_{k}\right)} \times\right. \\
& \left.\sum_{N \geq 1}(-1)^{N} \sum_{|[\mathbf{n}]|=N} \frac{\prod_{q=1}^{p} \Gamma\left(\theta_{k_{q}}+m_{q}+\alpha_{k_{q}}([\mathbf{n}])\right) \prod_{k \neq\left\{k_{1}, \ldots, k_{p}\right\}} \Gamma\left(\theta_{k}+\alpha_{k}([\mathbf{n}])\right)}{\Gamma(|\boldsymbol{\theta}|+m+2 N)} \prod_{k, l=1}^{K} \frac{\bar{W}_{k, l}^{n_{k, l}}}{n_{k, l} !}\right)
\end{aligned}
$$

where $\mathcal{Z}(\boldsymbol{\theta})$ is given by 42 ).

Considering the marginal event " $K_{1}=k_{1}, \ldots, K_{p}=k_{p} ; P_{K, m}=p$ ", it holds that

$$
\begin{aligned}
& \mathbf{P}\left(K_{1}=k_{1}, \ldots, K_{p}=k_{p} ; P_{K, m}=p\right) \\
= & \sum_{|\mathbf{m}|=m}^{\prime} \mathbf{P}\left(K_{1}=k_{1}, \ldots, K_{p}=k_{p} ; B_{K, m}\left(k_{1}\right)=m_{1}, . ., B_{K, m}\left(k_{p}\right)=m_{p} ; P_{K, m}=p\right),
\end{aligned}
$$

where the ${ }^{\prime}$-sum runs over all positive integers $\mathbf{m}=\left(m_{1}, . ., m_{p}\right)$ summing to $m$. There are $\left(\begin{array}{c}m-1 \\ p-1\end{array}\right)$ terms in this sum. And summing the latter probability over $k_{1}, \ldots, k_{p} \in\{1, \ldots, K\}^{p}$ gives $\mathbf{P}\left(P_{K, m}=p\right)$.

6.1.3. Generalized ESF in the 'Kingman $*$-limit', 23. Let $\theta_{k} \rightarrow 0$ for each $k=1, \ldots, K$ and $K \rightarrow \infty$ while $\sum_{k=1}^{K} \theta_{k}=|\boldsymbol{\theta}| \stackrel{*}{\rightarrow} \gamma>0$. We call it the $*$-limit and we wish to consider the generalized Ewens sampling formula in this limiting situation corresponding to a case with infinitely many alleles per locus, [33], [11, [39].

For instance, take $\theta_{k}=\theta / k, \sum_{k=1}^{K} \theta_{k}=\theta H_{K}$ where $H_{K}=\sum_{k=1}^{K} k^{-1}$, with $\theta \rightarrow 0$, $K \rightarrow \infty$ and $\theta \log K \rightarrow \gamma>0$.

With (48) and (49) mentioned below, we have

Theorem 6. In the $*$-limit, the probability of the occupancy event: " $K_{1}=k_{1}, \ldots, K_{m}=$ $k_{p} ; B_{m}\left(k_{1}\right)=m_{1}, . ., B_{m}\left(k_{p}\right)=m_{p} ; P_{m}=p$ ", with $m_{1}, . ., m_{p} \geq 1$ summing to $m$, exists and is given by

$$
\begin{aligned}
& \mathbf{P}\left(K_{1}=k_{1}, \ldots, K_{m}=k_{p} ; B_{m}\left(k_{1}\right)=m_{1}, . ., B_{m}\left(k_{p}\right)=m_{p} ; P_{m}=p\right) \\
& =\frac{\mathcal{Z}^{*}\left(\boldsymbol{\theta}+\mathbf{m}_{p}\right)}{\mathcal{Z}^{*}(\boldsymbol{\theta})}
\end{aligned}
$$

where $\mathcal{Z}^{*}\left(\boldsymbol{\theta}+\mathbf{m}_{p}\right)$ and $\mathcal{Z}^{*}(\boldsymbol{\theta})$ are given by (49) and (48) respectively.

Proof: In the $*$-limit, $1 / Z^{D}(\boldsymbol{\theta})=\Gamma(|\boldsymbol{\theta}|) / \prod_{k=1}^{K} \Gamma\left(\theta_{k}\right) \stackrel{*}{\sim} \Gamma(|\boldsymbol{\theta}|) \prod_{k=1}^{K} \theta_{k} \rightarrow 0$.

Consider first $\mathcal{Z}(\boldsymbol{\theta})$ and split the sum $\sum_{|[\mathbf{n}]|=N}$ into $\sum_{|[\mathbf{n}]|=N}^{1}:=\sum_{|[\mathbf{n}]|=N ; \alpha_{k}([\mathbf{n}])=0}$ and $\sum_{|[\mathbf{n}]|=N}^{2}:=\sum_{|[\mathbf{n}]|=N ; \alpha_{k}([\mathbf{n}]) \neq 0}$, where $\left\{\mathbf{n}:|[\mathbf{n}]|=N ; \alpha_{k}([\mathbf{n}])=0\right\}$ corresponds to those arrays $[\mathbf{n}]$ summing to $N$ and with $k$-th row and $k$-th column equal to 0 . We have

$$
\begin{aligned}
& \mathcal{Z}(\boldsymbol{\theta})=1+\frac{\Gamma(|\boldsymbol{\theta}|)}{\prod_{k=1}^{K} \Gamma\left(\theta_{k}\right)} \sum_{N \geq 1}(-1)^{N} \sum_{|[\mathbf{n}]|=N} \frac{\prod_{k=1}^{K} \Gamma\left(\theta_{k}+\alpha_{k}([\mathbf{n}])\right)}{\Gamma(|\boldsymbol{\theta}|+2 N)} \prod_{k, l=1}^{K} \frac{\bar{W}_{k, l}^{n_{k, l}}}{n_{k, l} !} \\
& =1+\frac{\Gamma(|\boldsymbol{\theta}|)}{\prod_{k=1}^{K} \Gamma\left(\theta_{k}\right)} \sum_{N \geq 1}(-1)^{N}\left(\sum_{|[\mathbf{n}]|=N}^{1} \frac{\prod_{k=1}^{K} \Gamma\left(\theta_{k}\right)}{\Gamma(\boldsymbol{\theta} \mid+2 N)} \prod_{k, l=1}^{K} \frac{\bar{W}_{k, l}^{n}, l}{n_{k, l} !}+\right. \\
& \left.+\sum_{|[\mathbf{n}]|=N}^{2} \frac{\prod_{k=1}^{K} \Gamma\left(\theta_{k}+\alpha_{k}([\mathbf{n}])\right)}{\Gamma(|\boldsymbol{\theta}|+2 N)} \prod_{k, l=1}^{K} \frac{\bar{W}_{k, l}^{n_{k, l}}}{n_{k, l} !}\right) \\
& \quad \stackrel{*}{\rightarrow} \mathcal{Z}^{*}(\boldsymbol{\theta}):=1+\frac{\Gamma(\gamma)}{\Gamma(\gamma+2 N)} \sum_{N \geq 1}(-1)^{N} \sum_{|[\mathbf{n}]|=N ; \alpha_{k}([\mathbf{n}])=0} \prod_{k, l=1}^{\infty} \frac{\bar{W}_{k, l}^{n_{k, l}}}{n_{k, l} !}
\end{aligned}
$$


Consider now $\mathcal{Z}\left(\boldsymbol{\theta}+\mathbf{m}_{p}\right)$ and split the sum $\sum_{|[\mathbf{n}]|=N}$ into the two parts $\sum_{|[\mathbf{n}]|=N}^{1}:=$ $\sum_{|[\mathbf{n}]|=N ; \alpha_{k}([\mathbf{n}])=0, k \neq\left\{k_{1}, \ldots, k_{p}\right\}}$ and $\sum_{|[\mathbf{n}]|=N}^{2}:=\sum_{|[\mathbf{n}]|=N ; \alpha_{k}([\mathbf{n}]) \neq 0, k \neq\left\{k_{1}, \ldots, k_{p}\right\}}$, where $\left\{\mathbf{n}:|[\mathbf{n}]|=N ; \alpha_{k}([\mathbf{n}])=0, k \neq\left\{k_{1}, \ldots, k_{p}\right\}\right\}$ corresponds to those arrays [n] whose entries sum to $N$ and with $k$-th row and $k$-th column equal to 0 , but only for those $k \neq\left\{k_{1}, \ldots, k_{p}\right\}$. We have

$$
\begin{aligned}
& \mathcal{Z}\left(\boldsymbol{\theta}+\mathbf{m}_{p}\right)=1+\frac{\Gamma(|\boldsymbol{\theta}|+m)}{\prod_{q=1}^{p} \Gamma\left(\theta_{k_{q}}+m_{q}\right) \prod_{k \neq\left\{k_{1}, \ldots, k_{p}\right\}} \Gamma\left(\theta_{k}\right)} \times \\
& \sum_{N \geq 1}(-1)^{N}\left(\sum_{|[\mathbf{n}]|=N}^{1} \frac{\left.\prod_{q=1}^{p} \Gamma\left(\theta_{k_{q}}+m_{q}+\alpha_{k_{q}}(\mathbf{n}]\right)\right) \prod_{k \neq\left\{k_{1}, \ldots, k_{p}\right\}} \Gamma\left(\theta_{k}\right)}{\Gamma(|\boldsymbol{\theta}|+m+2 N)} \prod_{k, l=1}^{K} \frac{\bar{W}_{k, l}^{n_{k, l}}}{n_{k, l} !}\right. \\
& \left.+\sum_{|[\mathbf{n}]|=N}^{2} \frac{\prod_{q=1}^{p} \Gamma\left(\theta_{k_{q}}+m_{q}+\alpha_{k_{q}}([\mathbf{n}])\right) \prod_{k \neq\left\{k_{1}, \ldots, k_{p}\right\}} \Gamma\left(\theta_{k}+\alpha_{k}([\mathbf{n}])\right)}{\Gamma(|\boldsymbol{\theta}|+m+2 N)} \prod_{k, l=1}^{K} \frac{\bar{W}_{k, l}^{n_{k}, l}}{n_{k, l} !}\right) \\
& \quad \stackrel{*}{\rightarrow} \mathcal{Z}^{*}\left(\boldsymbol{\theta}+\mathbf{m}_{p}\right):=1+\frac{\Gamma(\gamma+m)}{\Gamma(\gamma+m+2 N)} \sum_{N \geq 1}(-1)^{N} \\
& \quad \sum_{|[\mathbf{n}]|=N ; \alpha_{k}([\mathbf{n}])=0, k \neq\left\{k_{1}, \ldots, k_{p}\right\}} \frac{\prod_{q=1}^{p} \Gamma\left(m_{q}+\alpha_{k_{q}}([\mathbf{n}])\right)}{\prod_{q=1}^{p} \Gamma\left(m_{q}\right)} \prod_{k, l=1}^{\infty} \frac{\bar{W}_{k, l}^{n_{k, l}}}{n_{k, l} !} .
\end{aligned}
$$

The $*$-limit expression of the occupancy event generalizes the Ewens partition distribution in the infinitely-many-alleles population genetics model with symmetric selection/mutation, as studied in [13] and [17.

\subsubsection{Marginal distributions and frequency spectrum. Let}

$$
p_{k}\left(x_{k}\right):=\int d\left(\mathbf{x} \backslash x_{k}\right) p(\mathbf{x})
$$

be the $k$-th marginal of $\mathbf{X} \sim p(\mathbf{x})$ as given by (43). Then

$$
\frac{1}{K} \sum_{k=1}^{K} p_{k}(x) d x=\mathbf{E}\left(\frac{1}{K} \sum_{k=1}^{K} \mathbf{1}\left(X_{k} \in d x\right)\right),
$$

as the frequency spectrum, is the density of alleles at equilibrium in a neighborhood of $x$, see [9, 10]. It is a uniform mixture of the marginals $p_{k}(x)$ whose precise computation is possible but involved. Note that, from (45), the marginal moments of $X_{k}$ are known.

6.2. The house-of-cards mutations potential case. We finally consider sampling formulae when the multi-allelic population is at equilibrium and subject only to mutation driving forces.

If $V(\mathbf{x})$ is the mutation potential under the house of cards condition

$$
V(\mathbf{x})=\log \mathcal{W}_{M}(\mathbf{x}), \text { where } \mathcal{W}_{M}(\mathbf{x})=e^{-|\boldsymbol{\mu}|} \prod_{k=1}^{K} x_{k}^{\mu_{k}},
$$

then, with

$$
\begin{gathered}
Z_{N}^{D}(2 N|\boldsymbol{\mu}|)=\int_{\mathcal{S}_{K}} d \mathbf{x} \prod_{k=1}^{K} x_{k}^{2 N \mu_{k}-1}=\frac{\prod_{k=1}^{K} \Gamma\left(2 N \mu_{k}\right)}{\Gamma(2 N|\boldsymbol{\mu}|)}<\infty, \\
p_{N}(\mathbf{x})=\frac{1}{Z_{N}^{D}(2 N|\boldsymbol{\mu}|)} \prod_{k=1}^{K} x_{k}^{2 N \mu_{k}-1}, \mathbf{x} \in \mathcal{S}_{K}
\end{gathered}
$$

the Dirichlet distribution $D_{K}$ on the simplex $\mathcal{S}_{K}$, with parameters $2 N \mu_{k}, k=$ $1, \ldots, K$. 
When dealing with weak mutations, the allelic equilibrium distribution is

$$
p(\mathbf{x})=\frac{1}{Z^{D}(\boldsymbol{\theta})} \prod_{k=1}^{K} x_{k}^{\theta_{k}-1}, \mathbf{x} \in \mathcal{S}_{K},
$$

the asymmetric Dirichlet distribution $D_{K}(\boldsymbol{\theta})$ on the simplex $\mathcal{S}_{K}$, with parameters $\theta_{k}, k=1, \ldots, K$ (unless $\boldsymbol{\theta}^{\prime}=\theta \mathbf{1}^{\prime}$ for some common $\theta>0$, in which case $D_{K}(\boldsymbol{\theta})$ boils down to the symmetric Dirichlet distribution $D_{K}(\theta)$ ). When dealing with the potential solely arising from mutations (i.e. when avoiding selection), the allelic equilibrium probability distribution is thus the one of $\mathbf{S} \stackrel{d}{=} \mathbf{X} \sim D_{K}(\boldsymbol{\theta})$, corresponding to Dirichlet spacings. A much simpler situation than the previous one involving both mutation and selection but still not so obvious (9], section 5.10). We shall derive some sampling formulae in this context.

6.2.1. The frequency of a typical allele at equilibrium. Pick at random an allele from $\mathbf{S} \sim D_{K}(\boldsymbol{\theta})$. It has frequency $S_{k}$ with probability $S_{k}$ and so the frequency of a size-biased picked allele at equilibrium is

$$
f\left(S_{1}, \ldots, S_{K}\right)=\sum_{k=1}^{K} S_{k}^{2}
$$

an homogeneous functional of degree 2. Applying (ii) of Theorem 1

$$
\mathbf{E}\left(\sum_{k=1}^{K} S_{k}^{2}\right)=\frac{\Gamma(|\boldsymbol{\theta}|)}{\Gamma(|\boldsymbol{\theta}|+2)} \mathbf{E}\left(\sum_{k=1}^{K} T_{k}^{2}\right)=\frac{1}{|\boldsymbol{\theta}|(|\boldsymbol{\theta}|+1)} \sum_{k=1}^{K} \theta_{k}\left(\theta_{k}+1\right) .
$$

The variance of $\sum_{k=1}^{K} S_{k}^{2}$ requires the computation of the expected value of $f\left(S_{1}, \ldots, S_{K}\right)=$ $\left(\sum_{k=1}^{K} S_{k}^{2}\right)^{2}=\sum_{k=1}^{K} S_{k}^{4}+2 \sum_{1 \leq k_{1}<k_{2} \leq K} S_{k_{1}}^{2} S_{k_{2}}^{2}$, as an homogeneous functional of degree 4 , which is

$$
\begin{aligned}
& \frac{\Gamma(|\boldsymbol{\theta}|)}{\Gamma(|\boldsymbol{\theta}|+4)}\left[\sum_{k=1}^{K} \mathbf{E}\left(T_{k}^{4}\right)+2 \sum_{1 \leq k_{1}<k_{2} \leq K} \mathbf{E}\left(T_{k_{1}}^{2}\right) \mathbf{E}\left(T_{k_{2}}^{2}\right)\right] \\
= & \frac{\Gamma(|\boldsymbol{\theta}|)}{\Gamma(|\boldsymbol{\theta}|+4)}\left[\sum_{k=1}^{K} \frac{\Gamma\left(\theta_{k}+4\right)}{\Gamma\left(\theta_{k}\right)}+2 \sum_{1 \leq k_{1}<k_{2} \leq K} \frac{\Gamma\left(\theta_{k_{1}}+2\right)}{\Gamma\left(\theta_{k_{1}}\right)} \frac{\Gamma\left(\theta_{k_{2}}+2\right)}{\Gamma\left(\theta_{k_{2}}\right)}\right] .
\end{aligned}
$$

The full law of $\sum_{k=1}^{K} S_{k}^{2}$ could be obtained while considering $f\left(S_{1}, \ldots, S_{K}\right)=$ $\exp \left(\lambda \sum_{k=1}^{K} S_{k}^{2}\right)$. This functional is no longer homogeneous and $(i)$ of Theorem 1 should then be applied, with some combinatorics involved which we skip.

6.2.2. Smallest and largest allelic frequencies from $\mathbf{S} \sim D_{K}(\boldsymbol{\theta})$. We shall now use $(i)$ of Theorem 1 to compute the joint distribution of the largest and smallest allelic frequencies in a $D_{K}(\boldsymbol{\theta})$ distributed population at equilibrium. Suppose $1 \geq b>a \geq 0$ and consider the spacings' functional

$$
f\left(S_{1}, \ldots, S_{K}\right)=\prod_{k=1}^{K} \mathbf{1}\left(a<S_{k} \leq b\right) .
$$


Then $\mathbf{E} f\left(S_{1}, \ldots, S_{K}\right)=\mathbf{P}\left(S_{(K)}>a, S_{(1)} \leq b\right)$ is the required probability, assuming $S_{(1)}>\ldots>S_{(K)}$ to be the order statistics of $\left(S_{1}, \ldots, S_{K}\right)$. The case $a=0(b=1)$ gives the probability $\mathbf{P}\left(S_{(1)} \leq b\right)$, respectively $\mathbf{P}\left(S_{(K)}>a\right)$.

From statement $(i)$ of Theorem 1 indeed, the quantity

$$
\Gamma(|\boldsymbol{\theta}|) p^{-|\boldsymbol{\theta}|} \prod_{k=1}^{K} \mathbf{P}\left(a<T_{k}(p) \leq b\right)=\Gamma(|\boldsymbol{\theta}|) \prod_{k=1}^{K}\left[\frac{1}{\Gamma\left(\theta_{k}\right)} \int_{a}^{b} t^{\theta_{k}-1} e^{-p t} d t\right]
$$

interprets as the Laplace transform of $\mathbf{P}\left(S_{(K)}(t)>a, S_{(1)}(t) \leq b\right) t^{|\boldsymbol{\theta}|-1}$. Inverting this Laplace transform and putting $t=1$ yields $\mathbf{P}\left(S_{(K)}>a, S_{(1)} \leq b\right)$. From this, we obtain directly

$$
\mathbf{P}\left(S_{(K)}>a, S_{(1)} \leq b\right)=\frac{\Gamma(|\boldsymbol{\theta}|)}{\prod_{k=1}^{K} \Gamma\left(\theta_{k}\right)} *_{k=1}^{K} h_{\theta_{k}}(1),
$$

where $*_{k=1}^{K} h_{\theta_{k}}(1)$ is the $K$-fold convolution of the functions

$$
t \rightarrow h_{\theta_{k}}(t)=t^{\theta_{k}-1} \mathbf{1}(b \geq t>a), k=1, \ldots, K,
$$

evaluated at $t=1$. If $b=1$, (54) gives the tail probability distribution of $S_{(K)}$ whereas $a=1$ gives the probability distribution of $S_{(1)}$.

We now briefly show in outline that these tools are also useful in the computation of simple sampling formulae.

6.2.3. Sampling and the Dirichlet multinomial distribution. Let $\left(U_{1}, \ldots, U_{m}\right)$ be $m$ iid uniform throws on $\mathbf{S} \sim D_{K}(\boldsymbol{\theta})$. Let $\mathbf{B}:=\left(B_{1}, \ldots, B_{K}\right)$ be an integralvalued random vector which counts the number of visits to the different types of alleles in a $m$-sample. Hence, if $K_{l}$ is the allele type which the $l$-th trial meets, then $B_{k}:=\sum_{l=1}^{m} \mathbf{1}\left(K_{l}=k\right), k=1, \ldots, K$.

With $\sum_{k=1}^{K} m_{k}=m$ and $\mathbf{m}:=\left(m_{1}, \ldots, m_{K}\right)$, conditionally given $\mathbf{S}$, we have the multinomial distribution:

$$
\mathbf{P}(\mathbf{B}=\mathbf{m} \mid \mathbf{S})=\frac{m !}{\prod_{k=1}^{K} m_{k} !} \prod_{k=1}^{K} S_{k}^{m_{k}} .
$$

Averaging over $\mathbf{S}$ and applying (ii) of Theorem 1 to compute $\mathbf{E}\left(\prod_{k=1}^{K} S_{k}^{m_{k}}\right)$, we find

$$
\mathbf{P}(\mathbf{B}=\mathbf{m})=\mathbf{E P}(\mathbf{B}=\mathbf{m} \mid \mathbf{S})=\frac{m !}{\prod_{k=1}^{K} m_{k} !} \frac{\prod_{k=1}^{K}\left[\theta_{k}\right]_{m_{k}}}{[|\boldsymbol{\theta}|]_{m}},
$$

where $[\theta]_{m}:=\theta(\theta+1) \ldots(\theta+m-1), k \geq 1,(\theta)_{0}:=1$. This distribution is known as the Dirichlet multinomial distribution.

Applying Bayes formula, the posterior distribution of $\mathbf{S}$ given $\mathbf{B}=\mathbf{m}$ is determined by its density at a point $\mathbf{s}$ on the simplex $\mathcal{S}_{K}$ as

$$
f_{\mathbf{S}}(\mathbf{s} \mid \mathbf{B}=\mathbf{m})=\frac{\Gamma(|\boldsymbol{\theta}|+m)}{\prod_{k=1}^{K} \Gamma\left(\theta_{k}+m_{k}\right)} \prod_{k=1}^{K} s_{k}^{\left(\theta_{k}+m_{k}\right)-1}, \mathbf{s} \in \mathcal{S}_{K} .
$$


This shows, as is well-known, that $\mathbf{S} \mid \mathbf{B}=\mathbf{m} \stackrel{d}{\sim} D_{K}(\boldsymbol{\theta}+\mathbf{m})$, where $\boldsymbol{\theta}+\mathbf{m}=$ $\left(\theta_{1}+m_{1}, \ldots, \theta_{K}+m_{K}\right)$ is obtained by shifting $\boldsymbol{\theta}$. In particular

$$
\mathbf{E}\left(S_{k} \mid \mathbf{B}=\mathbf{m}\right)=\frac{\theta_{k}+m_{k}}{|\boldsymbol{\theta}|+m}, k=1, \ldots, K .
$$

6.2.4. Pòlya urn sequence. This suggests the following recursive approach to the sampling formula where successive samples are now drawn from the corresponding iterative posterior distributions. More specifically, let $\left(K_{1}, \ldots K_{m}\right) \in\{1, \ldots, K\}^{m}$ be the types of the successive alleles thus drawn. Then,

$$
\begin{gathered}
\mathbf{P}\left(K_{1}=k_{1}\right)=\mathbf{E}\left(\mathbf{P}\left(K_{1}=k_{1}\right) \mid \mathbf{S}\right)=\mathbf{E}\left(S_{k_{1}}\right)=\frac{\theta_{k_{1}}}{|\boldsymbol{\theta}|}, \\
\mathbf{P}\left(K_{2}=k_{2} \mid K_{1}\right)=\frac{\theta_{k_{2}}+\mathbf{1}\left(K_{1}=k_{2}\right)}{|\boldsymbol{\theta}|+1}, \ldots, \\
\mathbf{P}\left(K_{m}=k_{m} \mid K_{1}, \ldots, K_{m-1}\right)=\frac{\theta_{k_{m}}+\sum_{q=1}^{m-1} \mathbf{1}\left(K_{q}=k_{m}\right)}{|\boldsymbol{\theta}|+m-1} .
\end{gathered}
$$

The joint distribution of $\left(K_{1}, \ldots, K_{m}\right)$ reads

$$
\begin{aligned}
& \mathbf{P}\left(K_{1}=k_{1}, \ldots, K_{m}=k_{m}\right) \\
& =\frac{\theta_{k_{1}}}{|\boldsymbol{\theta}|} \prod_{q=1}^{m-1} \frac{\theta_{k_{q+1}}+\sum_{r=1}^{q} \mathbf{1}\left(k_{r}=k_{q+1}\right)}{|\boldsymbol{\theta}|+q}=\frac{\prod_{q=1}^{m}\left(\theta_{k_{q}}+\sum_{r=1}^{q-1} \mathbf{1}\left(k_{r}=k_{q}\right)\right)}{[\mid \boldsymbol{\theta}]_{m}} .
\end{aligned}
$$

The sequence $K_{1}, \ldots, K_{m}$ is a Pòlya urn sequence.

6.2.5. Asymptotics of the occupancy vector. The joint conditional generating function of the full occupancy vector $\mathbf{B}$ reads

$$
\mathbf{E}\left(\prod_{k=1}^{K} u_{k}^{B_{k}} \mid \mathbf{S}\right)=\left(\sum_{k=1}^{K} u_{k} S_{k}\right)^{m},
$$

which is homogeneous with degree $d=m$ allowing to compute $\mathbf{E}\left(\prod_{k=1}^{K} u_{k}^{B_{k}}\right)$. Further, with $\bar{T}_{k}:=T_{k} / \sum_{k=1}^{K} T_{k}, T_{k} \sim \operatorname{gamma}\left(\theta_{k}\right), k=1, \ldots, K$, as above, using independence between $\left(\bar{T}_{k}, k=1, \ldots, K\right)$ and $\sum_{k=1}^{K} T_{k} \sim \operatorname{gamma}(|\boldsymbol{\theta}|)$ and recalling $\left(\bar{T}_{k}, k=1, \ldots, K\right) \sim D_{K}(\boldsymbol{\theta})$

$$
\begin{aligned}
& \mathbf{E}\left(\prod_{k=1}^{K} u_{k}^{B_{k} / m}\right)=\frac{\Gamma(|\boldsymbol{\theta}|)}{\Gamma(|\boldsymbol{\theta}|+m)} \mathbf{E}\left[\left(\sum_{k=1}^{K} u_{k}^{1 / m} T_{k}\right)^{m}\right] \\
& \underset{m \uparrow \infty}{\sim} \frac{\Gamma(|\boldsymbol{\theta}|)}{\Gamma(|\boldsymbol{\theta}|+m)} \mathbf{E}\left[\left(\sum_{k=1}^{K} T_{k}\right)^{m}\left(1+\frac{1}{m} \sum_{k=1}^{K} \bar{T}_{k} \log u_{k}\right)^{m}\right] \\
& \underset{m \uparrow \infty}{\sim} \frac{\Gamma(|\boldsymbol{\theta}|)}{\Gamma(|\boldsymbol{\theta}|+m)} \mathbf{E}\left(\sum_{k=1}^{K} T_{k}\right)^{m} \mathbf{E}\left(\prod_{k=1}^{K} u_{k}^{\bar{T}_{k}}\right)=\mathbf{E}\left(\prod_{k=1}^{K} u_{k}^{\bar{T}_{k}}\right)=\mathbf{E}\left(\prod_{k=1}^{K} u_{k}^{S_{k}}\right),
\end{aligned}
$$

because $\mathbf{E}\left(\sum_{k=1}^{K} T_{k}\right)^{m}=\frac{\Gamma(|\boldsymbol{\theta}|+m)}{\Gamma(|\boldsymbol{\theta}|)}$. This shows that

$$
\mathbf{B} / m \stackrel{d}{\rightarrow} \mathbf{S} \text { as } m \rightarrow \infty .
$$

Note that, applying the strong law of large numbers (conditionally given $\mathbf{S}$ ), the above convergence in law also holds almost surely: the normalized occupancy vector $\mathbf{B}$ from an $m$-sample converges to $\mathbf{S}$ itself. 
6.2.6. ESF from equilibrium distribution driven solely by mutations. Firstly, with $q_{k}>-\theta_{k}$, as we already observed, it holds

$$
\mathbf{E}\left(\prod_{k=1}^{K} S_{k}^{q_{k}}\right)=\frac{\Gamma(|\boldsymbol{\theta}|)}{\Gamma\left(|\boldsymbol{\theta}|+\sum_{k=1}^{K} q_{k}\right)} \prod_{k=1}^{K} \frac{\Gamma\left(\theta_{k}+q_{k}\right)}{\Gamma\left(\theta_{k}\right)}=\frac{Z^{D}(\boldsymbol{\theta}+\mathbf{q})}{Z^{D}(\boldsymbol{\theta})},
$$

where $\frac{\Gamma\left(\theta_{k}+q_{k}\right)}{\Gamma\left(\theta_{k}\right)}=\left[\theta_{k}\right]_{q_{k}}$ if $q_{k}$ is an integer. Therefore, considering the probability of an occupancy event as in (46):

Proposition 7. With $m_{1}, . ., m_{p} \geq 1$ summing to $m$, the Ewens sampling formula under asymmetric mutations only is

$$
\begin{aligned}
& \mathbf{P}\left(K_{1}=k_{1}, \ldots, K_{p}=k_{p} ; B_{K, m}\left(k_{1}\right)=m_{1}, . ., B_{K, m}\left(k_{p}\right)=m_{p} ; P_{K, m}=p\right) \\
& =\mathbf{E}\left(\prod_{q=1}^{p} S_{k_{q}}^{m_{q}}\right)=\frac{Z^{D}\left(\boldsymbol{\theta}+\mathbf{m}_{p}\right)}{Z^{D}(\boldsymbol{\theta})}=\frac{\Gamma(|\boldsymbol{\theta}|)}{\Gamma(|\boldsymbol{\theta}|+m)} \prod_{q=1}^{p} \frac{\Gamma\left(\theta_{k_{q}}+m_{q}\right)}{\Gamma\left(\theta_{k_{q}}\right)}
\end{aligned}
$$

where $\frac{\Gamma\left(\theta_{k_{q}}+m_{q}\right)}{\Gamma\left(\theta_{k_{q}}\right)}=\left[\theta_{k_{q}}\right]_{m_{q}}$.

If we now consider the $*$-limit for mutations,

$$
\mathbf{E}\left(\prod_{q=1}^{p} S_{k_{q}}^{m_{q}}\right) \stackrel{*}{\sim} \frac{\Gamma(\gamma)}{\Gamma(\gamma+m)} \prod_{q=1}^{p} \theta_{k_{q}}\left(m_{q}-1\right) ! \stackrel{*}{\rightarrow} 0
$$

showing that there is no proper $*$-limit of the occupancy probability (59).

Remark (standard Ewens sampling formula): Suppose $\theta_{k}=\theta, k=1, \ldots, K$ (the symmetric Dirichlet mutation model). Owing to exchangeability of the alleles

$$
\begin{aligned}
& \mathbf{P}\left(B_{K, m}(1)=m_{1}, \ldots, B_{K, m}(p)=m_{p} ; P_{K, m}=p\right) \\
& =\left(\begin{array}{c}
K \\
p
\end{array}\right)\left(\begin{array}{c}
m \\
m_{1} \ldots m_{p}
\end{array}\right) \frac{\Gamma(|\boldsymbol{\theta}|)}{\Gamma(\boldsymbol{\theta} \mid+m)} \prod_{q=1}^{p} \frac{\Gamma\left(\theta+m_{q}\right)}{\Gamma(\theta)}
\end{aligned}
$$

where the occupancy vector is, say, over the first $p$ alleles of $\mathcal{S}_{K}$.

Consider the $*$-limit where $\theta \rightarrow 0, K \rightarrow \infty$ while $\theta K \rightarrow \gamma>0$, 23. Owing to $\left(\begin{array}{c}K \\ p\end{array}\right) \stackrel{*}{\sim} K^{p} / p !, \prod_{q=1}^{p} \theta_{k_{q}}=\theta^{p}$ et $(K \theta)^{p} \stackrel{*}{\sim} \gamma^{p}$, we get

$$
\mathbf{P}\left(B_{K, m}(1)=m_{1}, . ., B_{K, m}(p)=m_{p} ; P_{K, m}=p\right) \stackrel{*}{\rightarrow} \frac{m !}{p !} \frac{\gamma^{p}}{[\gamma]_{m}} \frac{1}{\prod_{q=1}^{p} m_{q}}
$$

which is formula (29) of [16, for example. In contrast with the asymmetric Dirichlet model, the symmetric Dirichlet model admits a proper $*$-limit occupancy probability. This is one of the many facets 6 of the standard ESF, see [16] and [11. $\diamond$

Coming back to (59) in the asymmetric Dirichlet case, this suggests to consider the following $* *$-limit: choose $\left\{\theta_{k}\right\}$ in such a way that $|\boldsymbol{\theta}|:=\sum_{k=1}^{K} \theta_{k} \rightarrow \gamma$ as $K \rightarrow \infty$, with none of the $\theta_{k} \rightarrow 0$. Such a limiting model for the mutation rates $\theta_{k}$ was considered in [19].

Examples are:

\footnotetext{
${ }^{6}$ In [16, the ESF (61) is called the first ESF. A second ESF rather deals with the occupancy vector $\mathcal{A}_{K, m}(i), i \in\{0, \ldots, m\}$, which counts the number of alleles in the $m$-sample with $i$ representatives.
} 
(i) $\theta_{k}=p^{k}, k=1, \ldots, K$, for some $p \in(0,1)$ with $\gamma=p /(1-p)$.

(ii) $\theta_{k}=k^{-2}, k=1, \ldots, K$ with $\gamma=\zeta(2)=\pi^{2} / 6$.

In the $* *$-limit, therefore

$$
\mathbf{E}\left(\prod_{q=1}^{p} S_{k_{q}}^{m_{q}}\right) \stackrel{* *}{\rightarrow} \frac{\Gamma(\gamma)}{\Gamma(\gamma+m)} \prod_{q=1}^{p} \frac{\Gamma\left(\theta_{k_{q}}+m_{q}\right)}{\Gamma\left(\theta_{k_{q}}\right)} .
$$

The asymmetric Dirichlet model has a proper $* *$-limit occupancy probability (59), as given by (62).

To summarize:

Theorem 8. Consider the $m$-sampling problem from $\mathbf{S} \sim D_{K}(\boldsymbol{\theta})$. With $m_{1}, . ., m_{p} \geq$ 1 summing to $m$ and $p \leq m \wedge K$, the probability of the occupancy event

$$
\text { " } K_{1}=k_{1}, \ldots, K_{p}=k_{p} ; B_{K, m}\left(k_{1}\right)=m_{1}, . ., B_{K, m}\left(k_{p}\right)=m_{p} ; P_{K, m}=p "
$$

is given by (59). From (61) there is no non-degenerate limit of this probability in the *-limit but there is one in the **-limit, given by (62).

6.2.7. Moments, marginal distributions and frequency spectrum. Recalling (58), the marginal moments of $S_{k}$ are $\mathbf{E}\left[S_{k}^{q_{k}}\right]=\frac{\Gamma(|\boldsymbol{\theta}|)}{\Gamma\left(|\boldsymbol{\theta}|+q_{k}\right)}\left[\theta_{k}\right]_{q_{k}}$. So $S_{k} \sim \operatorname{beta}\left(\theta_{k},|\boldsymbol{\theta}|-\theta_{k}\right)$ with marginal density $p_{k}\left(s_{k}\right):=\int d\left(\mathbf{s} \backslash s_{k}\right) p(\mathbf{s})$ equal to

$$
p_{k}(s)=\frac{\Gamma(|\boldsymbol{\theta}|)}{\Gamma\left(\theta_{k}\right) \Gamma\left(|\boldsymbol{\theta}|-\theta_{k}\right)} s^{\theta_{k}-1}(1-s)^{|\boldsymbol{\theta}|-\theta_{k}-1}, s \in(0,1) .
$$

This gives the empirical average of $\mathbf{S}$ in a neighborhood of $s$ as

$$
\mathbf{E}\left(\frac{1}{K} \sum_{k=1}^{K} \mathbf{1}\left(S_{k} \in d s\right)\right)=\frac{1}{K} \sum_{k=1}^{K} p_{k}(s) d s .
$$

Else, $f_{K}(s)=\frac{1}{K} \sum_{k=1}^{K} p_{k}(s)$, as the frequency spectrum, is the density of alleles at equilibrium in a neighborhood of $s$. It is a uniform mixture of $\operatorname{beta}\left(\theta_{k},|\boldsymbol{\theta}|-\theta_{k}\right)$ distributed rvs, $k=1, \ldots, K$. Near $s=\{0,1\}, f_{K}(s) \sim s^{\theta_{*}-1}$ and $f_{K}(s) \sim$ $(1-s)^{|\boldsymbol{\theta}|-\theta_{*}-1}$, where $\theta_{*}=\min (\boldsymbol{\theta})$ and $\theta^{*}=\max (\boldsymbol{\theta})$.

Acknowledgments: T. Huillet acknowledges partial support both from the "Chaire Modélisation mathématique et biodiversité" and the labex MME-DII Center of Excellence (Modèles mathématiques et économiques de la dynamique, de l'incertitude et des interactions, ANR-11-LABX-0023-01 project).

\section{REFERENCES}

[1] Barbour, A.D., Ethier, S.N. and Griffiths R.C. A transition function expansion for a diffusion model with selection. The Annals of Appl. Prob. Vol. 10, No. 1, 123-162, 2000.

[2] Bürger, R. "The mathematical theory of selection, recombination, and mutation," Wiley Series in Mathematical and Computational Biology. John Wiley \& Sons, Ltd., Chichester, 2000.

[3] Castilloux, A.M. and Lessard, S. The fundamental theorem of natural selection in Ewens' sense (case of many loci). Theor. Popul. Biol. 48(3), 306-315, 1995.

[4] Charlesworth, B. and Jain, K. Purifying selection, drift, and reversible mutation with arbitrarily high mutation rates. Genetics, 198(4), 1587-1602, 2014.

[5] Edalat, A. Shahshahani Gradients. http://sharif.edu/ ₹ phahyad/edalat.pdf. 
[6] Ethier, S.N. and Kurtz, T.G. "Markov processes. Characterization and convergence". John Wiley \& Sons, Inc., New York, 1986.

[7] Evans, S.N. Diffusions on the simplex from Brownian motions on hypersurfaces. Lecture Notes-Monograph Series. Institute of Mathematical Statistics. 35-48, 2003.

[8] Ewens, W.J. "Mathematical population genetics. I. Theoretical introduction," Second edition. Interdisciplinary Applied Mathematics, 27. Springer-Verlag, New York, 2004.

[9] Ewens, W.J. The sampling theory of selectively neutral alleles. Theoret. Population Biology 3, $87-112,1972$.

[10] Ewens, W.J. and Li, W.H. Frequency spectra of neutral and deleterious alleles in a finite population. J. Math. Biol. 10(2), 155-166, 1980.

[11] Feng, S. Diffusion processes and the Ewens sampling formula. Statistical Science, 31(1), 20-22, 2016.

[12] Griffiths, R.C. Allele frequencies with genic selection. J. Math. Biol. 17(1), 1-10, 1983.

[13] Handa, K. Sampling formulae for symmetric selection. Electronic Communications in Probability, 10, 223-234, 2005.

[14] Hermisson, J., Redner, O., Wagner, H. and Baake E. Mutation-Selection balance: Ancestry, Load and Maximum Principle. Theor. Pop. Biol., Volume 62, Issue 1, 9-46, 2002.

[15] Hofbauer, J. The selection mutation equation. J. Math. Biol. 1985, 23, 41-53, 1985.

[16] Huillet, T. Sampling formulae arising from random Dirichlet populations. Comm. in Stat.: Theory and Methods, 34(5), 1019-1040, 2005.

[17] Huillet, T. Ewens sampling formulae with and without selection. J. of Comput. and Appl. Math. 206, 755-773, 2007.

[18] Huillet, T. and Martinez, S. Sampling from finite random partitions. Meth. and Comp. in Appl. Prob., Vol. 5, Issue 4, 467-492, 2003.

[19] Huillet, T. and Martinez, S. Dirichlet-Kingman partition revisited. Far East J. of Theoretical Stat., 24(1), 1-33, 2008.

[20] Huillet, T. and Martinez, S. Discrete Evolutionary Genetics: Multiplicative Fitnesses and the Mutation-Fitness Balance. Appl. Math., Vol. 2 No. 1, 11-22, 2011.

[21] Karlin, S. Mathematical models, problems, and controversies of evolutionary theory. Bull. Amer. Math. Soc. (N.S.) Volume 10, No 2, 221-273, 1984.

[22] Kingman, J.F.C. "Mathematics of genetic diversity." CBMS-NSF Regional Conference Series in Applied Mathematics, 34. Society for Industrial and Applied Mathematics (SIAM), Philadelphia, Pa., vii+70 pp. ISBN: 0-89871-166-5, 1980.

[23] Kingman, J.F.C. "Poisson processes." Oxford Studies in Probability, no 3, Clarendon Press, Oxford, 1993.

[24] Li, W.H. Maintenance of genetic variability under mutation and selection pressures in a finite population. P.N.A.S., 74(6), 2509-2513, 1977.

[25] Li, W.H. Maintenance of genetic variability under the joint effect of mutation, selection and random drift. Genetics, 90(2), 349-382, 1978.

[26] O'Brien, P. A genetic model with mutation and selection. Math. Biosc. 73(2), 239-251, 1985.

[27] Okasha S. Fisher's fundamental theorem of natural selection - a philosophical analysis. The British Journal for the Philosophy of Science, 59(3), 319-351, 2008.

[28] Price, G.R. Fisher's fundamental theorem made clear. Ann. Hum. Genet., Lond., 36(2), 129140, 1972.

[29] Rannala, B. Stationary Allele Frequency Distributions. eLS, 2003.

[30] Shahshahani, S. A new mathematical framework for the study of linkage and selection. Mem. Amer. Math. Soc., 17, 1979.

[31] Svirezhev, Y.M. "Optimum principles in genetics". In: Studies on Theoretical Genetics, V. A. Ratner (Ed), 86-102, Novosibirsk, USSR Academy of Science, 1972.

[32] Tran, T.D.; Hofrichter, J. and Jost, J. The free energy method for the Fokker-Planck equation of the Wright-Fisher model. Preprint no 29, Max-Planck-Institut für Mathematik in den Naturwissenschaften Leipzig, 2015.

[33] Watterson, G.A. The stationary distribution of the infinitely-many neutral alleles diffusion model. J. of Appl. Prob., 13(4), 639-651, 1976.

[34] Watterson, G.A. Heterosis or neutrality?. Genetics, 85(4), 789-814, 1977.

[35] Watterson, G.A. An analysis of multi-allelic data. Genetics, 88(1), 171-179, 1978.

[36] Watterson, G.A. Motoo Kimura's use of diffusion theory in population genetics. Theor. Pop. Biol., 49(2), 154-188, 1996. 
[37] Wright, S. in "Genetics, Paleontology and Evolution", eds. Jepsen, G. L., Simpson, G. G. \& Mayr, E. (Princeton Univ. Press, Princeton, NJ), 365-389, 1949.

[38] Zeng, Z.B.; Tachida, H. and Cockerham, C. C. Effects of mutation on selection limits in finite populations with multiple alleles. Genetics, 122(4), 977-984, 1989.

[39] Zhou, Y. Asymptotic Theory for Three Infinite Dimensional Diffusion Processes. Open Access Dissertations and Theses. Paper 8514. McMaster University. 2014.

Laboratoire de Physique Théorique et Modélisation, CNRS-UMR 8089 et Université de Cergy-Pontoise, 2 Avenue Adolphe Chauvin, 95302, Cergy-Pontoise, France, E-mail: THIERRY.HUILLET@U-CERGY.FR 